\title{
Quantum Communication Assisted MAC Protocol
}

\author{
by \\ Steve R. Cloutier, SSM, CD, BASc

\begin{abstract}
A thesis submitted to the
Faculty of Graduate and Postdoctoral Affairs

in partial fulfillment of the requirements for the degree of
\end{abstract}

Master of Computer Science

Ottawa-Carleton Institute for Computer Science

Department of School of Computer Science

Carleton University

Ottawa, Ontario

April, 2015

(C) Copyright

Steve R. Cloutier, SSM, CD, 2015 
The undersigned hereby recommends to the

Faculty of Graduate and Postdoctoral Affairs acceptance of the thesis

\title{
Quantum Communication Assisted MAC Protocol
}

\author{
submitted by Steve R. Cloutier, SSM, CD, BASc \\ in partial fulfillment of the requirements for the degree of \\ Master of Computer Science
}

Dr Michel Barbeau, Thesis Supervisor

Dr. Evangelos Kranakis, Thesis Examiner

Stephane Soteg Some, External Examiner (U of O)

\begin{abstract}
Dr. Douglas Howe
Chair, Department of Computer Science

Ottawa-Carleton Institute for Computer Science

Department of School of Computer Science

Carleton University
\end{abstract}

April, 2015 


\section{Abstract}

Quantum mechanics has been around since the early 1900s and computers made their first appearance in the late 1930s. In the decades after that, the two came together to bring about quantum computing. A sub field of quantum computing is quantum communications, the ability to transmit a quantum state from one place to another. This communication takes place between two entities.

Communication over a classic network involves many entities each vying for network time. This is done through various network protocols, one of them being Medium Access Control (MAC). Although quantum computing is currently a one-to-one medium, it's properties can be used to assist the MAC protocol to enhance it's current abilities. In this thesis, we propose two different quantum communication assisted MAC protocols to achieve shared communication over a classic network. These protocols are based on quantum entanglement principles and contention-free collision avoidance algorithms. 
I would like to dedicate this thesis to my wife Janet. Without her tireless support throughout the process, and her editing assistance, this paper would have been much harder to accomplish. 


\section{Acknowledgments}

I would like to acknowledge my supervisor, Dr. Michel Barbeau for his patience and guidance in the realization of this thesis. His constructive criticism and friendly advice made this task easier to handle. My sincere thanks. 


\section{Table of Contents}

Abstract $\quad$ iii

Acknowledgments $\quad$ v

Table of Contents vi vi

List of Tables $\quad$ ix

List of Figures $\quad$ x

Nomenclature $\quad$ xi

1 Introduction $\quad 1$

1.1 Quantum Communication Assisted Media Access Control . . . . . . . 1

1.2 Qubits and Entanglement . . . . . . . . . . . . . . 2

1.3 Previous Quantum Based MAC Protocol . . . . . . . . . . . . . 3

1.4 Problem Statement . . . . . . . . . . . . . . . . . . . 4

1.5 New Quantum Communication Assisted MAC Protocol . . . . . . . . 4

1.6 Thesis Overview . . . . . . . . . . . . . . . . . . . . 6

2 Background $\quad 7$

2.1 Qubits ........................ 7

2.2 Quantum Entanglement . . . . . . . . . . . . . 8

2.2.1 Entangled States . . . . . . . . . . . . . . . 9

2.2.2 How it is Entangled . . . . . . . . . . . . . . . . . . . . 11

2.2.3 Qubit Measurement . . . . . . . . . . . . . 12

2.3 Quantum Teleportation . . . . . . . . . . . . . . . . 14 
3 Quantum Communications: A Literature Review 16

3.1 Quantum Entanglement . . . . . . . . . . . . . . . . 16

3.1 .1 Quantum Key Distribution . . . . . . . . . . . . . . 17

3.2 Quantum Information Transmission . . . . . . . . . . . . . 18

3.3 Quantum Networking . . . . . . . . . . . . . . . . . . . . . . . . 19

3.3 .1 MAC Assisted Protocol . . . . . . . . . . . . . . . . . 20

3.4 Conclusion . . . . . . . . . . . . . . . . . . . . . . . . . . 21

4 Proposed Quantum Assisted MAC Protocols 22

4.1 Communication Protocol Multi Qubit Entanglement - Collision Avoid-

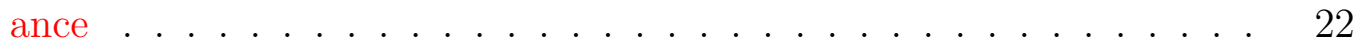

4.1 .1 One Client Station . . . . . . . . . . . . . . . . . 23

4.1 .2 Two Client Stations . . . . . . . . . . . . . . . . 23

4.1 .3 Three Client Stations . . . . . . . . . . . . . . . . . . 24

4.1.4 Four Client Stations . . . . . . . . . . . . . . . . 26

4.1 .5 Unused Time Slots . . . . . . . . . . . . . . . . . . . . . . 27

4.2 Communication Protocol Multi-Qubit Entanglement - Transmit First Election . . . . . . . . . . . . . . . . . . 27

4.2 .1 One Client Station . . . . . . . . . . . . . . . . 28

4.2 .2 Two Client Stations . . . . . . . . . . . . . . . . 28

4.2 .3 Three or More Client Stations . . . . . . . . . . . . . . . 29

4.2.4 AP Tasks and Joining Transmission Queue . . . . . . . . . 29

4.3 Summary . . . . . . . . . . . . . . . . . . . . 30

5 Protocol Testing and Assessment 31

5.1 Non-Contention Based Comparison . . . . . . . . . . . . . . 31

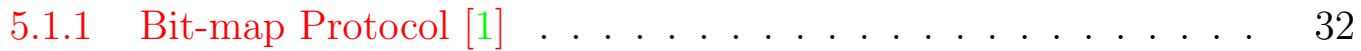

5.1.2 Binary-countdown Assignment Protocol [1] . . . . . . . . . 33

5.1.3 Assigned Transmission Frequencies Protocol . . . . . . . . . 34

5.1.4 Non-Contention Based Comparison Results . . . . . . . . . . . 34

5.2 Contention Based Simulation Comparison . . . . . . . . . . . 34

5.2.1 Qubit Entanglement Simulation . . . . . . . . . . . . . 35

5.2 .2 Architecture . . . . . . . . . . . . . . . . 35

5.2 .3 Event Based . . . . . . . . . . . . . . . . . . . 36

5.2 .4 Protocol Testing . . . . . . . . . . . . . . . 37 
5.2.5 Contention Based Simulation Comparison Results . . . . . . . 38

5.2.6 Statistical Analysis of Data . . . . . . . . . . . . . . . . 42

6 Conclusions and Future Work 45

$\begin{array}{ll}\text { List of References } & 48\end{array}$

$\begin{array}{ll}\text { Appendix A Acronyms } & 50\end{array}$ 


\section{List of Tables}

4.1 Two CS Qubit Transmit Number Combinations . . . . . . . . . . . 24

4.2 Three CS Qubit Combinations . . . . . . . . . . . . . 25

4.3 Two CS Qubit Combinations . . . . . . . . . . . . . . 28

4.4 Four CS Qubit Combinations . . . . . . . . . . . . . 29

4.5 Total Time-slots used Protocol Comparisons . . . . . . . . . . . . 30

5.1 Binary Count Down Protocol . . . . . . . . . . . . . . . 33

5.2 Transmission Efficiency Comparisons . . . . . . . . . . . . . . 41

5.3 Calculated Transmission Efficiencies For Other Numbers of CSs . . . 42

5.4 F-Test Aloha vs TFE . . . . . . . . . . . . . . . . . . 43

$5.5 \quad$ F-Test Aloha vs CA . . . . . . . . . . . . . . . . . 43

5.6 T-Test Aloha vs TFE . . . . . . . . . . . . . . . . 44

5.7 T-Test Aloha vs CA . . . . . . . . . . . . . . . . . 44 


\section{List of Figures}

1.1 Seven Layer OSI Model . . . . . . . . . . . . . . . . . . . . 2

1.2 Qubit Entanglement Abstraction . . . . . . . . . . . . 3

1.3 Typical Collision Avoidance Frame With Three CSs . . . . . . . 5

1.4 Typical Transmit First Election Frame With Four CSs . . . . . . . 5

2.1 Hong-Ou-Mandel Effect . . . . . . . . . . . . . . . . . . . 11

2.2 Photon Polarization $|0\rangle \ldots \ldots \ldots \ldots \ldots$

2.3 Photon Polarization $|1\rangle \ldots \ldots \ldots \ldots$

2.4 Classic Facsimile . . . . . . . . . . . . . . . . . . . . 15

2.5 Quantum Teleportation . . . . . . . . . . . . . . . 15

4.1 Transmission Cycle With One CS . . . . . . . . . . . . . . 23

4.2 Cycle and CS Transmit Position With Two CSs . . . . . . . . . 24

4.3 Qubit Pair Distribution to Three CSs . . . . . . . . . . . 24

4.4 Cycle and CS Transmit Position With Three CSs . . . . . . . 25

4.5 Qubit Triad Distribution to Four CSs . . . . . . . . . 26

4.6 Cycle and CS Transmit Position With Four CSs . . . . . . . . 27

4.7 Transmission Cycle With One CS . . . . . . . . . . . . . 28

4.8 Cycle and CS Transmit Position With Two Remaining CSs . . . . . 29

4.9 Total Transmission Cycle With Four CSs . . . . . . . . . . . 30

5.1 Bit-map Contention Slot Transmission Intention . . . . . . . . . . 32

5.2 Bit-map Transmission Slots . . . . . . . . . . . . . . . . . . 32

5.3 Architecture of Collision Avoidance Network _ . . . . . . . 36

5.4 Architecture of Transmit First Election Network . . . . . . . . 37

5.5 Aloha Simulation With 4 Hosts . . . . . . . . . . . . 38

5.6 Collision Avoidance Simulation With 4 Hosts . . . . . . . . . . 38

5.7 Aloha Simulation With 6 Hosts . . . . . . . . . . . . . . 39

5.8 Transmit First Election Simulation With 6 Hosts . . . . . . . . . 39

5.9 Transmission Efficiency for Aloha Protocols . . . . . . . . . . . 41 


\section{Nomenclature}

\section{Notation}

This thesis uses the following Dirac's ket notation:

$|0\rangle,|1\rangle$ Basis vectors for a qubit in the computational (Z) basis.

$|+\rangle,|-\rangle$ Basis vectors for a qubit in the $\mathrm{X}$ basis.

$|\psi\rangle$ Notation for a state vector. Generic for the state vector of a pure state of one or more qubits. It may be either a physical qubit or a logical one encoded using quantum error correction, depending on the context.

$\left|\Phi^{ \pm}\right\rangle,\left|\Psi^{ \pm}\right\rangle$(AB) A Bell pair with one qubit held by node A and one qubit held by node B. 


\section{Chapter 1}

\section{Introduction}

Quantum mechanics appeared around the beginning of the 20th century. Shortly after computers came about in the late 1930s, ideas of quantum computing came into being. This in turn led to two subsections of quantum computer science, those being quantum computing and quantum communication. Quantum communication, unlike quantum computing has moved from the theoretical world into the real world with some of its ideas. Quantum communication is the ability to transmit a quantum state from one place to another. This communication event takes place between two entities. On the other hand classic wireless communication allows many Client Stations (CS) to communicate with each other. The control of the access to the medium is handled by a Medium Access Control (MAC) protocol. Quantum communication is a one-to-one communication medium, however it's properties can be exploited to assist a MAC protocol. A quantum assisted MAC protocol is first proposed by Berces and Imre [2].

\subsection{Quantum Communication Assisted Media Ac- cess Control}

The internal functions of a computer communication system is characterized into abstraction layers in a conceptual model called the Open Systems Interconnection (OSI) model. This model consists of seven layers as shown in Figure 1.1. These layers handle different functions and resources within the communication process. The layer we are interested in here, is the data link layer. This layer provides the node-to-node connect-ability and consists of two sub layers, the Logical Link Control layer and the Media Access Control (MAC) layer. The focus of this thesis is the MAC 
layer.

Figure 1.1: Seven Layer OSI Model

\begin{tabular}{|c|c|}
\hline 7 & Application \\
\hline 6 & Presentation \\
\hline 5 & Session \\
\hline 4 & Transport \\
\hline \hline 3 & Network \\
\hline 2 & Data Link \\
\hline 1 & Physical \\
\hline
\end{tabular}

Within this thesis we examine two network protocols that we have developed for a quantum assisted MAC protocol that implement a contention-free collision avoidance technique. These protocols are similar to classic algorithms but involve the concept of qubits and exploiting quantum entanglement using two or more entangled qubits.

\subsection{Qubits and Entanglement}

In classical digital computing a bit is the basic unit of information. This bit can only be one of two values, either "0" or "1". With quantum computing we have the quantum bit, which is more commonly referred to as a qubit. Similar to the bit, a qubit can be measured in two states known as basis states. With a bit it is either a 0 or 1 , whereas with a qubit it can be a $|0\rangle$ or a $|1\rangle$ (read as ket 0 and ket 1$)$. There is also an important difference, similar to a coin toss, the value of the qubit cannot be determined until it is actually measured, and only one value is measured. For example, when a coin is tossed in the air, while it is spinning it is either heads or tails. Not until it is caught and looked at, is its value of heads or tails determined. The same as a qubit, not until it is measured can it be determined whether it is a $|0\rangle$ or a $|1\rangle$.

Quantum entanglement is a property where in a group of entangled qubits (e.g. 2 to 14), each qubit directly reflects the measurement of the other qubits. At the time of entanglement, there is no determination as to what the value of the qubits 
are. The value of the qubits are not determined until at least one of them is actually measured. The resultant value of each of the entangled qubits depends on the number of entangled qubits and which entanglement state is used. The most common entanglements are Bell, W, and Greenberger Horne Zeilinger (GHZ) states. To entangle these qubits, it is usually done on the subatomic level by creating direct interactions between particles. For a two qubit entanglement using a Bell state, it can be likened to a coin toss by two people as in Figure 1.2. Two people each flip a coin that are magically entangled. Without looking at the outcome they put them into a box and go their separate ways. At some point one of them looks into his or her own box to see what the coin is (either a head or a tail). From this point on whenever the other person looks at their coin, it will be the opposite (either a tail or a head). Similarly, in a Bell pair entangled state, one qubit is always a $|0\rangle$ and the other is a $|1\rangle$.

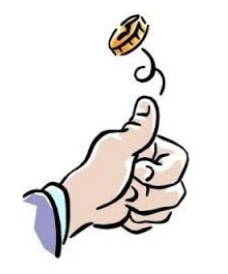

Qubit A

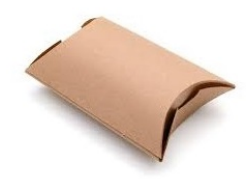

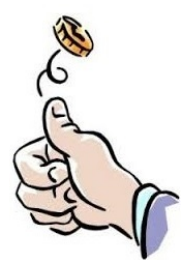

Qubit B

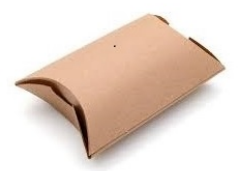

Figure 1.2: Qubit Entanglement Abstraction

\subsection{Previous Quantum Based MAC Protocol}

Quantum based MAC protocol is not a new concept. In 2006 Berces and Imre [2], proposed a quantum assisted MAC protocol. They suggest using a quantum channel access sublayer and a MAC sublayer, within the MAC layer, to track access requests. One half of a Bell state entangled qubit pair is sent to the two nodes requesting access. Whichever node gets the $|0\rangle$ transmits in the next time slot. This algorithm only works for two nodes requesting access to the medium. Several years later Arizmendi et al. [3] 
also propose adaptation of quantum communication in a 802.11 MAC protocol. In this case they propose sending using a combination of an Einstein Podolsky Rosen (EPR) entangled pair to determine who transmits and a $\mathrm{W}$ state entangled qubit triplet to control transmission. This proposal also only works in cases where there are only two CSs wishing to transmit. The protocols put forth in this thesis work for an arbitrary number of CSs.

\subsection{Problem Statement}

In this thesis we study how quantum communication can be exploited to assist a MAC protocol. Solutions proposed in the past, work for networks of only two CSs. The problem is to develop a solution that works with an arbitrary number of CSs. We put forth two such solutions that work with arbitrary numbers of CSs.

\subsection{New Quantum Communication Assisted MAC Protocol}

Both protocols we put forth are contention-free and based on a cycle of Time Slots (TS) which enable all the CSs present to have equal and fair access to the medium. The number of TSs in a typical cycle depends on the number of CSs present that wish to transmit and the protocol used. A standard TS for transmission of a packets is set to $100 \mathrm{~ms}$. The TS for transmission of entangled qubits by the Access Point (AP) is set to $5 \mathrm{~ms}$, to allow for transmission of up to 48 entangled qubits and monitoring of CS transmission requests. When a new CS transmission request comes in, the AP includes that CS to the next cycle. If a CS has not transmitted for two cycles, it is dropped from further cycles until it requests to transmit again.

The first protocol is strictly Collision Avoidance(CA). This protocol works for one to four CSs. The number of TSs needed in one cycle is 2 for 1 CS, 3 for 2 CSs, 8 for 3 CSs and 16 for 4 CSs. An example of a cycle for three CSs is shown in Figure 1.3. For this example, in TS1, the AP prepares the required number of entangled bits using the appropriate state, and transmits these to the three CSs wishing to transmit. It also monitors if there are any new CSs wishing to transmit. During TS2 to TS8, the three CSs read their received entangled qubits and use these to calculate their 
transmit number. This transmit number determines which slot they transmit in. At this point the cycle begins again with all the CSs wishing to transmit included.

Figure 1.3: Typical Collision Avoidance Frame With Three CSs

\begin{tabular}{|c|c|c|c|c|c|c|c|}
\hline $\mathrm{AP}$ & \multicolumn{7}{|c|}{$\Longleftarrow$ CS Tx slots $\Longrightarrow$} \\
\hline TS1 & TS2 & TS3 & TS4 & TS5 & TS6 & TS7 & TS8 \\
\hline
\end{tabular}

The second protocol is a Transmit First Election (TFE) type collision avoidance protocol. This protocol works for any number of CSs. The number of TSs needed for a complete cycle is $2 \mathrm{n}-1$, where $\mathrm{n}$ is the number of CSs present wishing to transmit. Figure 1.4 shows a cycle in the case where there are four CSs transmitting. For this example, in TS1, the AP prepares the four entangled qubits using W state, and transmits these to the four CSs wishing to transmit. It also monitors if there are any new CSs wishing to transmit. When received, the four CSs read their entangled qubit. The CS that receives a $|1\rangle$ transmits in TS2. In TS3, the AP prepares three entangled qubits using $\mathrm{W}$ state, and transmits these to the remaining three CSs wishing to transmit. It also monitors if there are any new CSs wishing to transmit. When received, the three CSs read their entangled qubit. The CS that receives a $|1\rangle$ transmits in TS4. In TS5, the AP prepares two entangled qubits using W state, and transmits these to the remaining two CSs wishing to transmit. It also monitors if there are any new CSs wishing to transmit. When received, the two CSs read their entangled qubit. The CS that receives a $|1\rangle$ transmits in TS6 and the CS that receives a $|0\rangle$ transmits in TS7. At this point the cycle begins again with all the CSs wishing to transmit included.

Figure 1.4: Typical Transmit First Election Frame With Four CSs

\begin{tabular}{|c|c|c|c|c|c|c|}
\hline AP & 1st CS & AP & 2nd CS & AP & 3rd CS & 4th CS \\
\hline TS1 & TS2 & TS3 & TS4 & TS5 & TS6 & TS7 \\
\hline
\end{tabular}




\subsection{Thesis Overview}

In the next chapter, we give a brief overview of quantum communications and some of the main ideas needed to further comprehend this study. Chapter 3 gives a literature review of quantum entanglement, quantum information transmission, and quantum networking. This chapter also touches on some writings that explain where we stand with quantum communication technology and network capabilities. In Chapter 4, we give an in depth explanation of our two proposed protocols and what is expected to happen when implemented. The simulation run of the two protocols and a comparison to current protocols is discussed in Chapter 5 . Chapter 6 covers some of the limitations involved in using quantum communication in a multiuser network and the future work needed to advance this topic. Also in Chapter 6, is our conclusions on quantum communication use in a multiuser network is discussed. 


\section{Chapter 2}

\section{Background}

The idea of quantum mechanics appeared around the beginning of the 20th century. Throughout that century and into the next, this idea has expanded to other fields of science. The field of computer science was not to be left out of this quantum concept. In the 1970s and 1980s, there was an increase of interest in the study of quantum systems, especially ideas related to creating a quantum state, dynamical operations, and simulating classical conventions. This in turn led to two subsections of quantum computer science, those being quantum computing and quantum communication. Quantum computing is still in its infancy, studying how the quantum-mechanical phenomena can be theoretically used in computational systems. Quantum communication however has moved from the theoretical world into the real world with some of its ideas.

Quantum communication is the ability to transmit a quantum state from one place to another. There have been many recent developments in the field of Quantum Com-

munication, from the number of qubits that can be entangled together, the distance that these qubits can be transmitted both with and without wires, and network implementations. To further understand Quantum Communications we first review a few key concepts that are used within the proposed network protocols. Although there are many aspects of quantum communication, the two main concepts that are related to this paper are that of the qubit and quantum entanglement.

\subsection{Qubits}

With classical digital computing a bit is the basic unit of information. This bit can only be one of two values, either "0" or "1". With quantum computing we have 
the Quantum Bit, which is more commonly referred to as a qubit. Similar to the bit, a qubit can be measured in two states known as basis states (or basis vectors). In quantum computing these states are written using Dirac or bra-ket notation and appear as $|0\rangle$ and $|1\rangle$ (pronounced ket 0 and ket 1). The similarities end there. With a bit it is either a 0 or 1 , whereas with a qubit, it can be in a state other than $|0\rangle$ or $|1\rangle$, and can be represented as a linear combination of states referred to as superpositions (see Formula 2.1), where $\alpha$ and $\beta$ are probability amplitudes and can in general both be complex numbers [4].

$$
|\psi\rangle=\alpha|0\rangle+\beta|1\rangle,
$$

Similar to Schrödingers Cat [5], the value of the qubit cannot be determined until it is actually measured. We measure the qubit in the standard basis and obtain the probability of outcome $|0\rangle$ is $|\alpha|^{2}$ and the probability of outcome $|1\rangle$ is $|\beta|^{2}$. Since the absolute squares of the amplitudes equate to probabilities, $\alpha$ and $\beta$ must fall within the Formula 2.2. This ensures that either one state or the other is measured.

$$
|\alpha|^{2}+|\beta|^{2}=1
$$

\subsection{Quantum Entanglement}

Quantum entanglement is a property where in a group of entangled qubits (two to fourteen), each qubit directly reflects the measurement of the other qubits [6]. When the qubits in this group are entangled, they are reflective of each other. At the time of entanglement, there is no determination as to what the value of the qubits are. The value of the qubits are not determined until at least one of them is actually measured. When one of the qubits in an entangled group is measured, the rest of the group is determined at the same time. For example if two qubits are entangled and one is measured as a $|1\rangle$ the other qubit in the pair is automatically a $|0\rangle$. If one is measured as a $|0\rangle$ the other in the pair is a $|1\rangle$. Different outcomes to these measurements depends on the number of qubits and the state that is used to entangle them. This phenomenon, "Spooky Action at a Distance" as it was referred to by Einstein, occurs regardless of the distance between the qubits at the time of measuring, whether it is a few centimeters or thousands of kilometers. 


\subsubsection{Entangled States}

Throughout the years of research, theories and experiments refer to different states of entanglement. The most common ones are Bell state, W state, and Greenberger Horne Zeilinger (GHZ) state.

Bell State [4]: In the case of two entangled qubits, one method is referred to as a Bell state (see Formula 2.3). In this state there are two qubits entangled as a pair. The pair can be split with one qubit given to one person and the other given to another, but they are entangled to each other.

$$
\left|\Phi^{-}\right\rangle=\frac{1}{\sqrt{2}}\left(|0\rangle_{A} \otimes|0\rangle_{B}-|1\rangle_{A} \otimes|1\rangle_{B}\right)
$$

This equation is broken down as follows: The first qubit (subscript A) can be a 0 or 1 . When this qubit is measured by $A$, the outcome is random, with either outcome being possible with the probability of $1 / 2$. Then if $B$ measures their qubit, the outcome would be the same as A. Therefore B would be a random outcome as well. If $\mathrm{A}$ and $\mathrm{B}$ communicate they see that there is a correlation between their results.

Based on quantum mechanics, we can see that the qubits can be in quantum superposition, a 0 and 1 simultaneously. (see Formula 2.4).

$$
|+\rangle=\frac{1}{\sqrt{2}}(|0\rangle+|1\rangle) \text { or }|-\rangle=\frac{1}{\sqrt{2}}(|0\rangle-|1\rangle)
$$

If $\mathrm{A}$ and $\mathrm{B}$ choose to measure on this basis, to check whether their qubits were $|+\rangle$ or $|-\rangle$ for instance, they find the same correlations as previous, because the Bell state can be formally rewritten as follows (Formula 2.5):

$$
\left|\Phi^{+}\right\rangle=\frac{1}{\sqrt{2}}\left(|+\rangle_{A} \otimes|+\rangle_{B}+|-\rangle_{A} \otimes|-\rangle_{B}\right)
$$

There are four possible outcomes in this state, the one explained above, and 3 other combinations. All together they are known as the four maximally entangled two-qubit states (or just Bell States) (see Formulas 2.6, 2.7, 2.8, and 2.9).

$$
\left|\Phi^{-}\right\rangle=\frac{1}{\sqrt{2}}\left(|0\rangle_{A} \otimes|0\rangle_{B}-|1\rangle_{A} \otimes|1\rangle_{B}\right)
$$




$$
\begin{aligned}
& \left|\Phi^{+}\right\rangle=\frac{1}{\sqrt{2}}\left(|0\rangle_{A} \otimes|0\rangle_{B}+|1\rangle_{A} \otimes|1\rangle_{B}\right) \\
& \left|\Psi^{-}\right\rangle=\frac{1}{\sqrt{2}}\left(|0\rangle_{A} \otimes|1\rangle_{B}-|1\rangle_{A} \otimes|0\rangle_{B}\right) \\
& \left|\Psi^{+}\right\rangle=\frac{1}{\sqrt{2}}\left(|0\rangle_{A} \otimes|1\rangle_{B}+|1\rangle_{A} \otimes|0\rangle_{B}\right)
\end{aligned}
$$

In this state when one is measured as a $|1\rangle$ the other qubit in the pair is automatically a $|0\rangle$. If one is measured as a $|0\rangle$ the other in the pair is a $|1\rangle$.

W State [7]: The W State comprises of two or more entangled qubits. See Formula 2.10 for the case of three entangled bits.

$$
|W\rangle=\frac{1}{\sqrt{3}}(|001\rangle+|010\rangle+|100\rangle)
$$

It has been theorized that $\mathrm{W}$ state can be generalized for $\mathrm{n}$ qubits and thus the quantum superpostion has equal expansion coefficients of all possible pure states in which exactly one of the qubits in an "excited state" $|1\rangle$, while all other ones are in the "ground state" $|0\rangle$ (see Formula 2.11).

$$
|W\rangle=\frac{1}{\sqrt{n}}(|100 \ldots 0\rangle+|010 \ldots 0\rangle+\ldots+|000 \ldots 1\rangle)
$$

Greenberger Horne Zeilinger State [8]: In this state there are three qubits entangled. Greenberger Horne Zeilinger (GHZ) state is based on the same principles as the Bell state. Because of this, when we condense the formulas, for a system of $n$ qubits the GHZ state we can write it as (Formula 2.12):

$$
|G H Z\rangle=\frac{|0\rangle^{\otimes n}+|1\rangle^{\otimes n}}{\sqrt{2}}
$$

As a simple example using a 3-qubit GHZ state we have the following (Formula 2.13):

$$
|G H Z\rangle=\frac{1}{\sqrt{2}}(|000\rangle+|111\rangle)
$$


The three qubits can be split and given to each one of a group of three, but they are entangled to each other. However, in this state when one qubit is read as a $|1\rangle$, the other two are as well. If one is read as a $|0\rangle$, the other two are as well. So in this entanglement state the outcome of the three qubits are either $|000\rangle$ or $|111\rangle$.

\subsubsection{How it is Entangled}

To entangle these qubits, it is usually done on the subatomic level by creating direct interactions between particles. This can be done in numerous ways such as spontaneous parametric down-conversion or Hong-Ou-Mandel effect [9].

Spontaneous Parametric Down Conversion Spontaneous Parametric Down Conversion (SPDC) is the process where photons are split into pairs using a nonlinear crystal. This split pair has correlated polarizations and are phase-matched in the frequency domain. This happens due to the law of conservation of momentum and the law of conservation of energy. The resulting pair either has the same polarization (Type I) or perpendicular polarization (Type II). This method results in random creations of entangled particles and therefore has very low conversion efficiency.

Hong-Ou-Mandel Effect If you send a photon to a beam splitter it either is transmitted or reflected. The reflectivity of the beam splitter determines its relative probability of transmission and reflection. For the Hong-Ou-Mandel Effect, we assume 50:50 beam splitter, meaning there is an equal probability that the photon is transmitted or reflected.
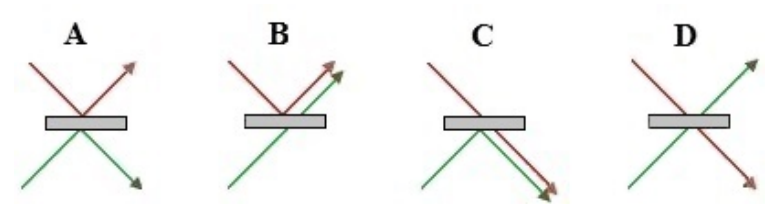

Figure 2.1: Hong-Ou-Mandel Effect

If you send two photons to the 50:50 beam splitter at the same time, one to each of input mode, there are four possible outcomes (see Figure 2.1). The four results are: 
- A) The top photon (shown here in red) is reflected and the bottom (shown in green) is also reflected;

- B) The top photon is reflected and the bottom is transmitted;

- C) The top photon is transmitted and the bottom is reflected;

- D) The top photon is transmitted and the bottom is also transmitted;

This produces two photons that are identical in their physical properties of polarization, spatio-temporal mode structure and frequency. Both beams start $180^{\circ}$ out of phase to each other. Transmission does not change the original phase. If both photon are transmitted as in D, they remain equal and opposite and cancel each other out. Reflection off the splitter causes a relative phase shift of $-90^{\circ}$ for the bottom and $+90^{\circ}$ at the top. In A, since they are both phase shifted $90^{\circ}$ the result ends up canceling themselves. Therefore only B and C produce usable entangled photon pairs. Since at this point they are $90^{\circ}$ out of phase with each other the resultant difference can be measured.

\subsubsection{Qubit Measurement}

As stated previously there are various ways to create and entangle qubits. It is only logical that there are various ways to measure a qubit. The type of measurement used depends on the way the qubit was created, or the method of entanglement use. The two main methods of measurement use Polarization or Spin to determine the value of the qubit.

Polarization In its basic form a photon can be represented as a electromagnetic wave. This wave has an oscillating electric field which orients to either the y (horizontal) as in Figure 2.2 or $\mathrm{x}$ (vertical) axis as in Figure 2.3. The measurement of this orientation determines the value of the qubit, which gives us a $|0\rangle$ or a $|1\rangle$. 
CHAPTER 2. BACKGROUND

13

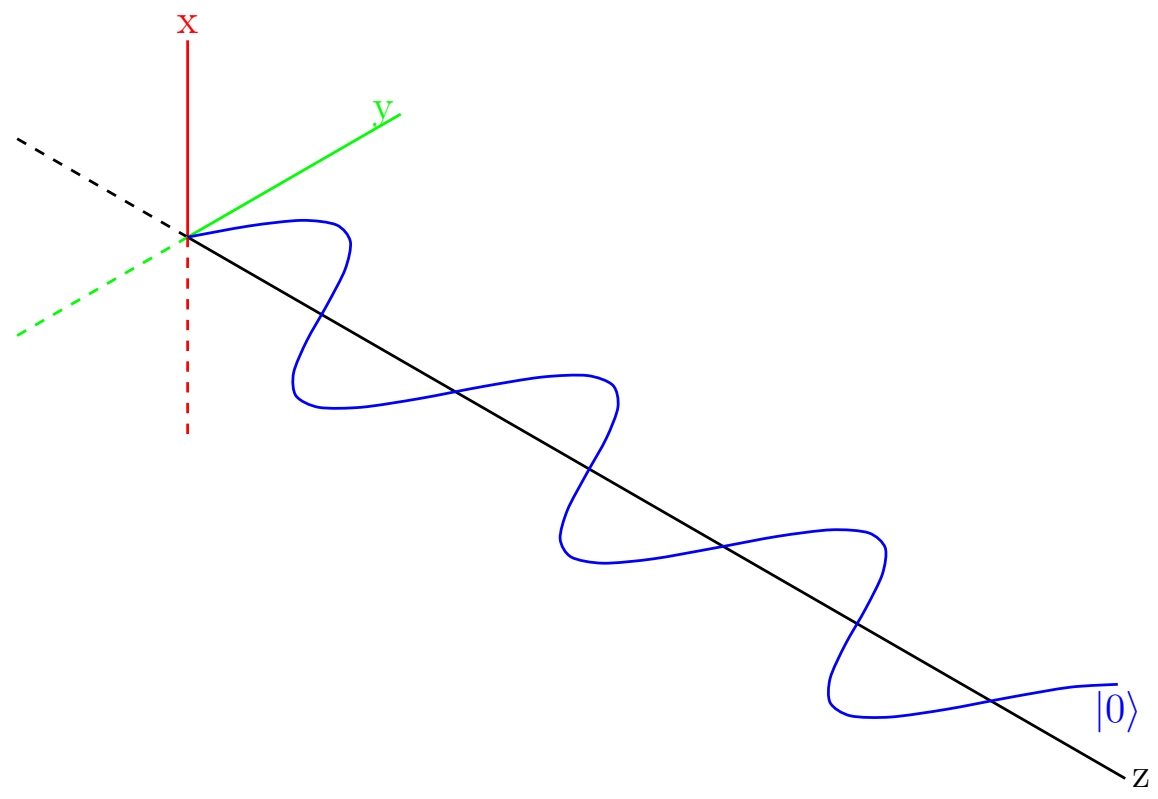

Figure 2.2: Photon Polarization $|0\rangle$

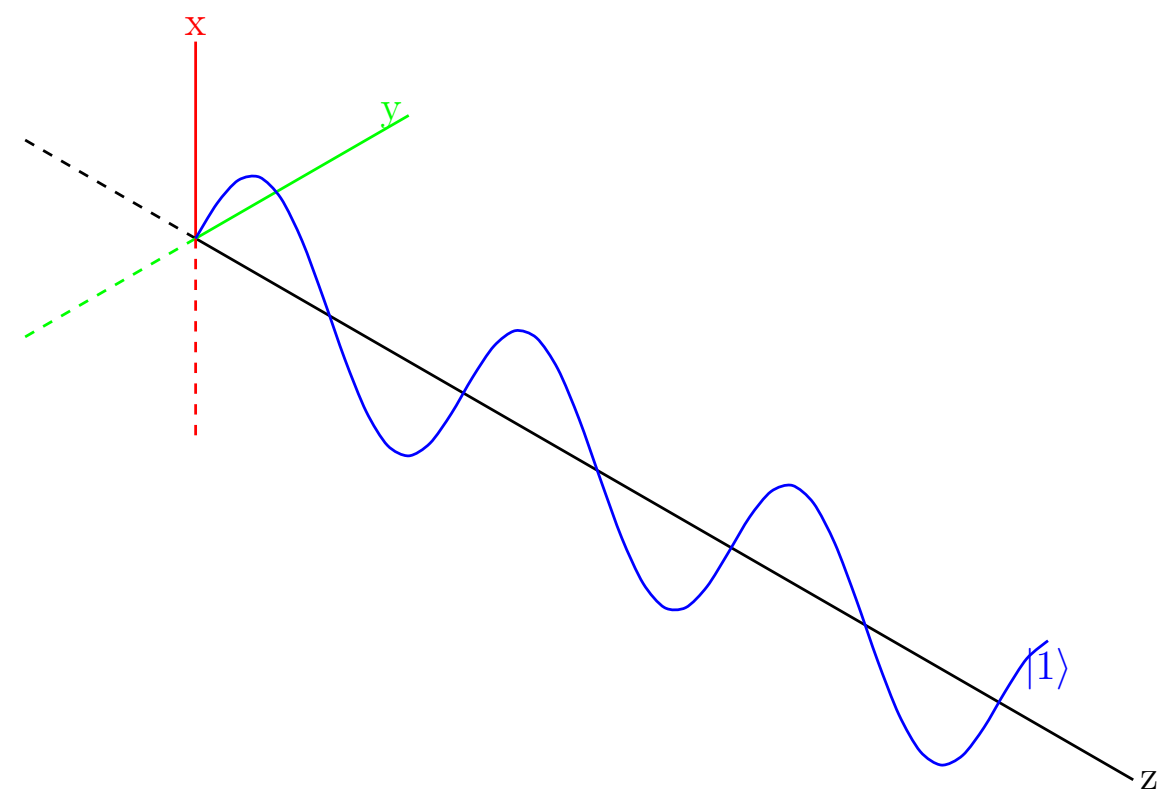

Figure 2.3: Photon Polarization $|1\rangle$ 
Spin There are many different mechanical means to measure the spin of the qubit and thus its value. The basic principle is that the spin of a particle is a two state system which is the magnetic moment of an electron. It is either a clockwise rotation (spin-up) which is interpreted as a $|1\rangle$, or a counter clockwise rotation (spindown) which is interpreted as a $|0\rangle$.

\subsection{Quantum Teleportation}

Quantum Teleportation is the process used to transmit quantum information from source to destination using classical communication [6]. This method is used in instances where the equipment being used or distance being covered is not acceptable to qubit transmitting. The exact state of the information (atom or proton) is transmitted using previously shared quantum entanglement and classical communication. The information contained in the qubit is transmitted from location to location and not the actual physical qubit. This process can be compared to transmitting a facsimile as in Figure 2.4. The information on the original document is transmitted to the destination while the original document remains at the source.

The process for transmitting the information in a qubit is done this similar way as shown in Figure 2.5. The information contained in the original qubit is transmitted, and not the qubit itself. 


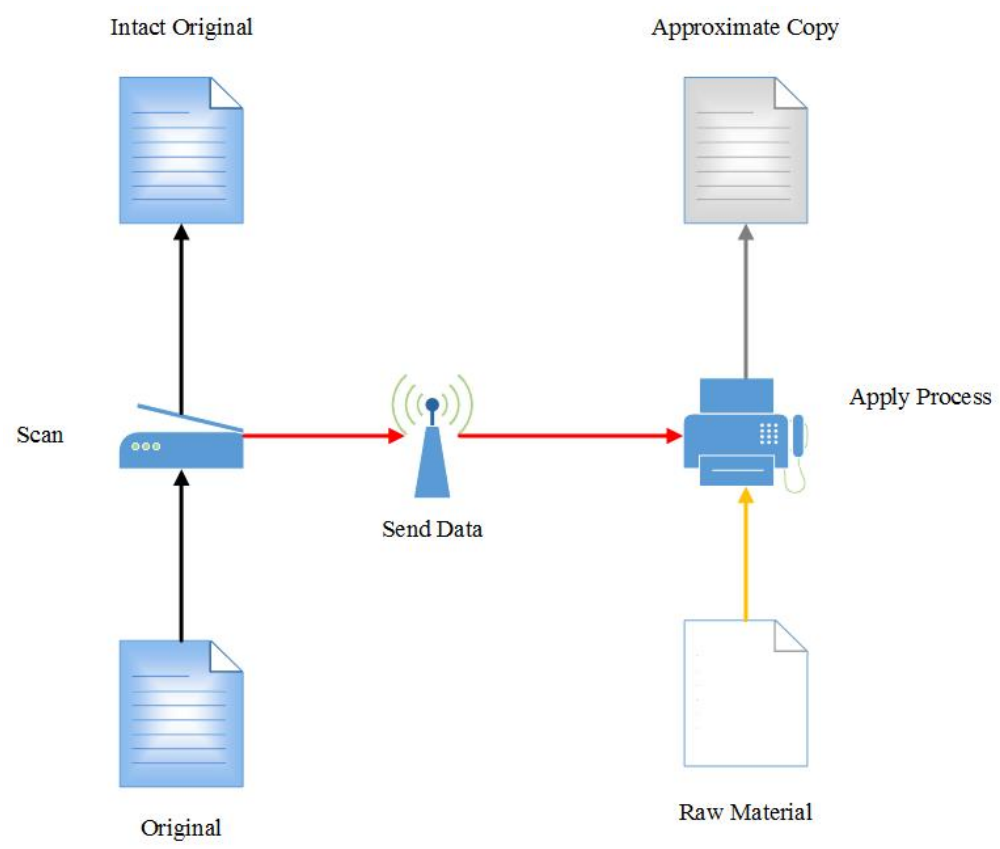

Figure 2.4: Classic Facsimile

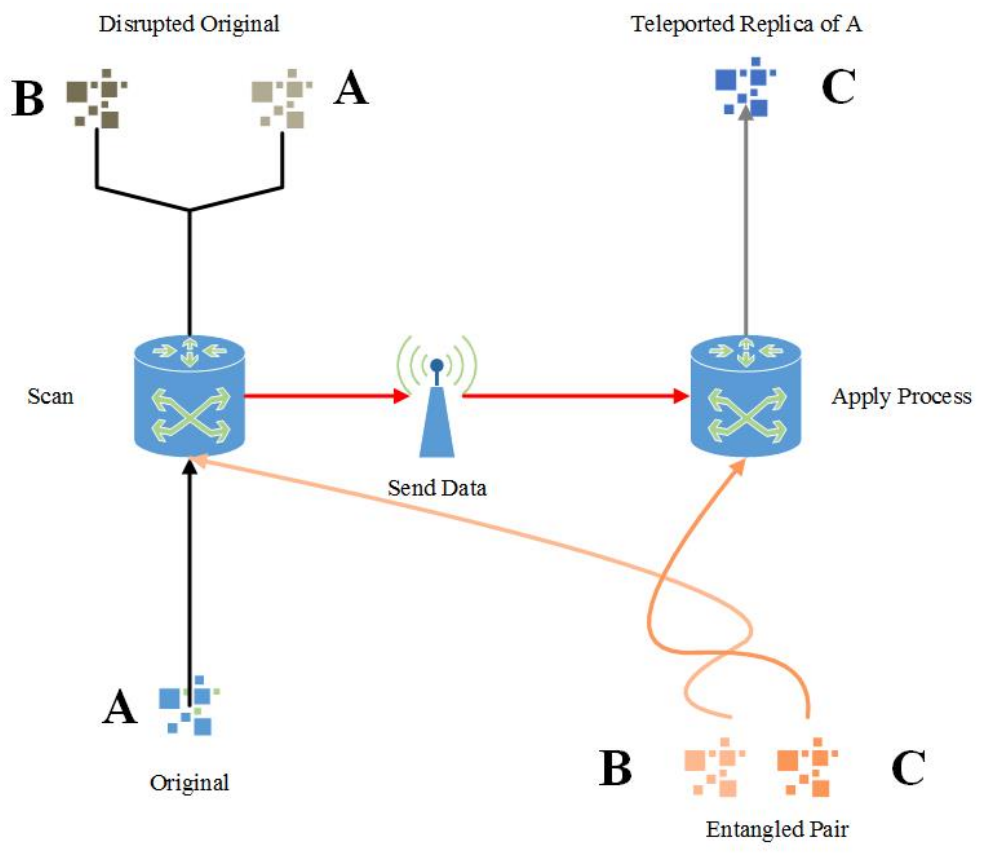

Figure 2.5: Quantum Teleportation 


\section{Chapter 3}

\section{Quantum Communications: A Literature Review}

Quantum communication is the ability to transmit a quantum state from one place to another. There are many developments in the field of quantum communication from the number of qubits that can be entangled together to the distance that these qubits can be transmitted both with and without wires. These developments lead to quantum key distribution, quantum teleportation, and quantum networking. In this chapter we review some the most important papers that show where quantum communication is today, and how we got there. These papers relate to the topic that is addressed within this thesis.

\subsection{Quantum Entanglement}

Quantum communication is based on a theory developed by Einstein et al. [10] in 1935 about using a quantum-mechanical description of physical reality in the real world. This would then be known as the Einstein Podolsky Rosen Paradox or just EPR. In 1964, Bell [11] used this EPR pair to put forth a theory of what the EPR pairs are. Over the years, this theory has been expanded and proven to become what we now know as the Bell State pair. These Bell State pairs (explained in Chapter Two) are the foundation of quantum entanglement.

What about entanglement of more than two qubits? In 2000, Dur et al. [7] shows how entangling three qubits can produce two inequivalent results. Their paper defines equivalence classes of entangled states by the use of invertible local transformations of a multipartite system. Classification of the entanglement properties of the state 
is compared. This comparison determines if two states that have the same kind of entanglement can be derived from each other using Local Operations and Classical Communication (LOCC). This method shows two inequivalent types of tripartite entanglement, when comparing three-qubit systems of pure states, especially the GHZ and $\mathrm{W}$ states. It is determined that a $\mathrm{W}$ state has maximally bipartite entanglement in this case.

Since we can entangle two or three qubits, why not four? Verstraete et al. [12] showed in 2002 that entangling four qubits can be done in nine different ways. Using the principal of Stochastic Local quantum Operations assisted by Classical Communication (SLOCC) they investigate a single copy of a pure four-partite state of qubits. Doing so, allows them to classify all the different states of the four qubits. They are able to show that entangling four qubits can be done in nine different ways, and thus nine families of states. The various states are GHZ like, and two or three qubit entanglement distributed among the four parties. A seven parameter family of entanglement monotones is developed using concurrence and tri-entanglement applied to the states of four qubits. An optimal protocol is produced using the SLOCC operations maximizing the entanglement monotones.

In 2011 Monz et al. [13] shows us that 14-qubit entanglement is possible. This paper is a report on the largest number of qubits entangled so far, that being 14 . This entanglement is achieved using GHZ states. By investigating the decay of coherent ions over time, they show a correctness with a theoretical model to prove their premise.

\subsubsection{Quantum Key Distribution}

Secure communication within quantum computing can be achieved using quantum mechanics to produce Quantum Key Distribution (QKD). This QKD allows two users to share secret data in an encryption like state. The level of security achieved is at far higher levels than that possible with classic computer encryption. Not only is this encryption virtually unbreakable, but because of the principles of this encryption, it is also possible to detect if someone tried to eavesdrop on an encrypted transmission.

Bennett et al. [14] first brought about this theory in 1984. Their theory shows that using quantum systems, such as polarized photons, it is possible to transmit digital data that is impossible in principal to eavesdrop without a high probability of

being detected. Random key information is distributed between two users through a quantum channel in conjunction with classic channels, to ensure that it remains 
unknown to anyone else. In classic public-key cryptography the data shared between two users is concealed from a passive eavesdropper by use of trapdoor functions, even though there is no initial shared secret information between those two users. In QKD, random bits that contain no information are transmitted between the two users. Through further consultation, over a classic channel using a BB84 protocol, can detect with high probability if the original data has been disturbed in transit by an eavesdropping third party, and they have received the same bits. If no tampering occurred, then they can agree that these random bits can be used as a One Time Pad (OTP) to conceal subsequent communications.

In a Tokyo QKD Network, Sasaki and a host of others [15] recently successfully tested quantum key distribution. This group put into place a secure quantum encrypted network using GHz-clocked QKD systems, a QKD smart-phone, a reliable commercial QKD product, and an entanglement QKD system. They made use of the OTP to securely distribute data between users. An OTP allows secure transmissions for the current session. If the session is terminated, then the whole QKD process has to be repeated to establish a new OTP. Their demonstration showed that practical use of quantum encryption in a full size network is not far off.

As recently as 2015, Mailloux et al. [16] put forth a paper that shows the state of QKD today. Although the original BB84 protocol still works well, with more interest in this type of security, it has started to become vulnerable to attacks. To protect from this, QKD systems need to be protected by use of controlled telecom environments. With further development of transmission technology, QKD can still make for very secure communications.

\subsection{Quantum Information Transmission}

Communicating classically can be done using wireless transmission over great distances, however doing the same using quantum devices has been an issue. The main hindrances to doing this has been signal dissipation, strength, and line of sight problems. The ability to extend this over larger distances, and even globally is of considerable interest to the field. Ursin et al. [17] reports on an experiment that shows that a quantum transmission can be done at a distance of $144 \mathrm{~km}$. The experiment was carried out using polarized entangled photon pairs produced in a type-II parametric down-conversion source, a $\beta$-barium-borate crystal, and a high-power ultraviolet 
laser. One photon of the entangled pair was measured at the transmission site, and the other was received and read $144 \mathrm{~km}$ away over a free-space optical link by use of a $15 \mathrm{~cm}$ transceiver and a $1 \mathrm{~m}$ mirror telescope receiver. Previous distances of up to $13 \mathrm{~km}$ [18] were reached using polarization-entangled photons via free-space links through the atmosphere. Continued progress in this endeavor is necessary to lead to future satellite-based quantum communication.

The process of transferring quantum states between two separate atoms is known as teleportation or quantum teleportation. Quantum teleportation depends on the properties of entanglement and the use of classic communication to make it work (as discussed in chapter two). Quantum teleportation was first theorized by Bennett et al. [19]. The fact that transmissions of entangled bits over great distances is not possible with the technology of the day, other methods need to be used. With the use of entanglement properties and classic communication, the state of the bit can be teleported over greater distances than that of standard qubit transmission technology. As reported by Singhal et al [20] in their investigative paper, teleportation can provide so called invisible quantum wiring to achieve ultra-fast computers. These new quantum computers would be able to compute larger and more complex loads at many times the speed of today's super computers.

\subsection{Quantum Networking}

As stated in chapter one and two, advancing quantum communication needs the ability to create a quantum network. In the last few years there has been a lot of attention given to this endeavor. Van Meter is one person who is leading the way in developing this technology. To start off, Van Meter et al. [21], explain how developing the quantum network has some of the same issues that are encountered in developing classic network. They also believe that some of the scalability and management issues in developing the Internet would have been solved in a more uniform fashion if quantum communication was used thus avoiding the redundant engineering effort that occurred. To avoid the same issue with the quantum repeater network development their proposal can be used with all existing repeater design. They present an idea for a Quantum Recursive Network Architecture, based on the extension of classic recursive networks [22]. This recursive network represents a subset of the network as a single node at a different layer of that network. Their architecture proposal uses 
arbitrary distributed states, such as Bell pairs, GHZ, W, and cluster states to set up the repeater network between nodes.

To build on the idea of the quantum repeater network, Van Meter teams up with Aparicio and Esaki [23] to propose the needed protocol design for such a network. Quantum repeater networks necessitate classical protocols to handle quantum operations. In this paper they implement their own protocols. This involves state machines and packet sequencing that use a three protocol layer and management of resources. The quantum State propagation layer (Entanglement Swapping Control)is the first layer. Administering the Bell pairs is handled within this layer. The second layer, link entanglement control (ACKed Entangled Control), is responsible for handling the single-hop physical entanglement process. The third layer is the error management layer (purification control). This layer chooses two Bell pairs, and elects one pair to have its fidelity boosted and the other to be sacrificed, assuring that both stations make the same decisions. This three layer stack consists of ACKed Entangled Control, Purification Control (PC), and Entanglement Swapping Control (ESC). The PC and ESC layers can be combined recursively to span arbitrary numbers of hops.

In 2012, Van Meter further develops quantum networking and internetworking [24]. Internetworking encompasses connecting multiple disparate networks. Multiple different technologies and many different management domains coexist in any large network that grows and evolves over time forming an internetwork. Communication systems are having new capabilities using quantum networks based on quantum measurement and entanglement. Teleportation and quantum effects improve the networks and allow for computation over increasing distances. Due to some of the inherent issues in quantum operations, assertive and cooperative management is necessary amongst nodes. Development into large scale types of this network is increasing with theoretical and experimental work which will make it a reality in the coming years.

\subsubsection{MAC Assisted Protocol}

A big part of quantum networking is the ability to integrate quantum properties into Medium Access Control (MAC). To wireless telecommunication systems, MAC protocols are important and integral. These protocols allow for the users to best take advantage of common resources. The communication channel is a part of these resources. In 2006, Berces and Imre [2] show how it is possible to use quantum methods 
to model MAC. It is shown that algorithms and calculations can become more efficient using quantum informatics computation. Benefits can be seen by implementing these protocols into existing classical networks. This also assists in the developing of the MAC protocols for future quantum networks.

To further delve into this topic, in 2012 Arizmendi et al. [3] show how a quantum communications adaptation of $802.11 \mathrm{MAC}$ protocol is theoretically possible. The proposed protocol can be used for both classic and quantum communications, and ad hoc or base station type networks. To get a high data rate and avoid collisions, they make use of quantum entanglement and quantum parallelism. This protocol uses a MAC sublayer for the classic communications.

\subsection{Conclusion}

As can be seen, Research and Development (R\&D) of quantum communications has been increasing exponentially over the years. Continuing in this direction could ultimately lead to full quantum networks complementing classic networks in the coming years. This $R \& D$ in quantum communication is also a huge step in the right direction to advance the field of quantum computers. 


\section{Chapter 4}

\section{Proposed Quantum Assisted MAC Protocols}

As proposed, these protocols only assist the Media Access Control (MAC) protocol. They are used only to determine which CS has the right to transmit. When this has been done, the actual transmission is done using classic wireless protocol. Two different protocols are proposed here similar to classic algorithms, using either two or three entangled qubits to achieve communication within a network. One protocol is based on collision avoidance, while the other is more of a leader election type protocol.

\subsection{Communication Protocol Multi Qubit Entan- glement - Collision Avoidance}

This protocol achieves 100\% collision avoidance. This is done using Bell state entangled qubit pairs and/or W-State entangled qubit triads. The number of Time Slots (TS) within one cycle varies depending on the number of Client Stations (CS) wishing to transmit. This allows optimal time for users to transmit as well as to join the communication cycle with minimal wait times. For this protocol, the TS for packet transmission is $100 \mathrm{~ms}$. This gives sufficient time for a CS to transmit a packet of data of 952 bits at a transmission rate of 9600 bps. The TS for the Access Point (AP) to transmit entangled qubits is set at $5 \mathrm{~ms}$. This gives the sufficient time to transmit the required entangled qubits as well as to monitor which CSs are present who wish to transmit. (The time of $5 \mathrm{~ms}$ is chosen for simulation and comparison. In reality the time is determined depending on the method of qubit entanglement transmission, such as photon or teleportation.) 
During the first time slot in each cycle, the AP monitors the traffic to see if there are requests to transmit from any new CS and if a present CS has been idle for more than two cycles. If a present CS has been idle they are dropped from the next cycle, and have to request again to join any subsequent cycles. Entering and exiting protocol is based on existing Time Division Multiple Access (TDMA) protocol.

\subsubsection{One Client Station}

If the AP determines that there is only one CS, then that CS transmits in TS2, and then the process repeats again from the beginning. Refer to Figure 4.1.

Figure 4.1: Transmission Cycle With One CS

\begin{tabular}{|c|c|}
\hline 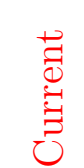 & 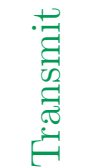 \\
\hline$\Downarrow$ & $\Downarrow$ \\
\hline TS1 & TS2 \\
\hline
\end{tabular}

\subsubsection{Two Client Stations}

If the AP has determines that there are only two CSs wishing to transmit, it then sends a pair of entangled bits to them. One half of the pair to each of them. When they receive their qubit, they read them. Based on the principles of entangled pairs, one reads a zero, the other reads a one. This is their Transmit Number (TN). All possible combinations of two entangled qubits are shown in Table 4.1. This TN lets each of them know when to transmit. If the CS reads a zero for the TN, they transmit in the next TS. If they read a one for their TN, they skip the next TS and transmit in the one after that. At the completion of the TSs (which would be three TSs in this case as in Figure 4.2), the cycle repeats at the beginning again. 
Table 4.1: Two CS Qubit Transmit Number Combinations

\begin{tabular}{|c|c|}
\hline CS1 & CS2 \\
\hline 1 & 0 \\
\hline 0 & 1 \\
\hline
\end{tabular}

Figure 4.2: Cycle and CS Transmit Position With Two CSs

\begin{tabular}{|c|c|c|}
\hline 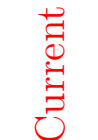 & $\underbrace{x}_{0}$ & $E_{-1}^{x}$ \\
\hline$\Downarrow$ & $\Downarrow$ & $\Downarrow$ \\
\hline TS1 & $\mathrm{TS} 2$ & TS3 \\
\hline
\end{tabular}

\subsubsection{Three Client Stations}

Upon determining that there are three CSs wishing to transmit, the AP sends two entangled qubit pairs to each of the CSs. They each receive one half of a pair that another one receives for a total of three sets of pairs as shown in Figure 4.3.

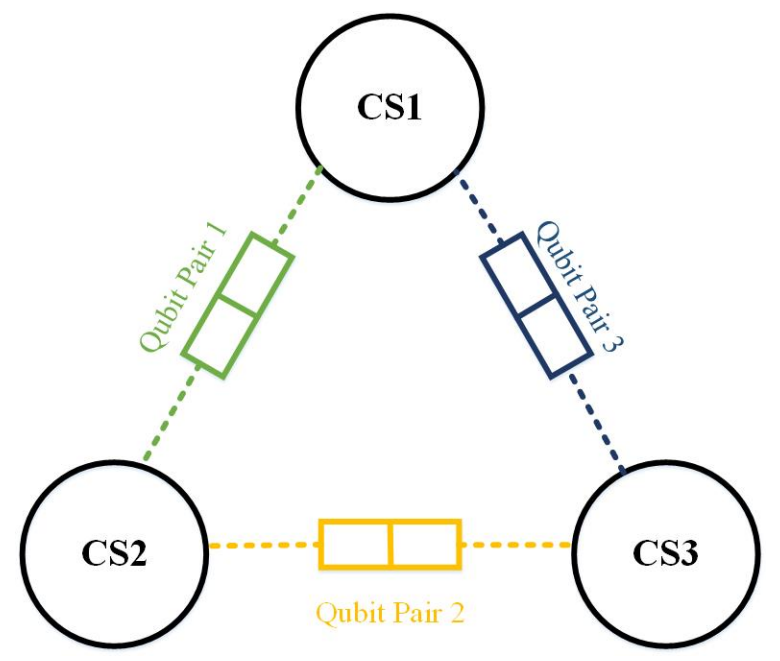

Figure 4.3: Qubit Pair Distribution to Three CSs

As per the entangled protocol, when each pair is read, one side is a one, and the other a zero. The three qubit pairs have a number associated with them from one to three. When the CS reads this qubit, it puts the result in the that position of a 
3 qubit binary number, and then puts a zero in the empty slot. For example, CS1 receives one qubit from pair one, which goes in it's position one, and the second from qubit pair three which goes in the third position. The empty position number two receives a zero. (All possible combinations are shown in Table 4.2.) This leaves the CS with a three qubit binary TN that is unique to itself. This TN tells it how many TSs to wait until it transmits. For example if they have a zero they transmit in the next slot. If they have a 110 they transmit in the sixth slot after the next one. See Figure 4.4. Since the "111" combination does not exist, only seven transmission slots are available. In each cycle there are four slots where a CS does not transmit. These slots can be used by the AP for other tasks (covered later in this paper).

Table 4.2: Three CS Qubit Combinations

\begin{tabular}{|c|c|c|l|}
\hline CS1 & CS2 & CS3 & \\
\hline 101 & 000 & 010 & \\
101 & 010 & 000 & \\
100 & 000 & 011 & Qubit Pair 1 \\
100 & 010 & 001 & Qubit Pair 2 \\
000 & 100 & 011 & Qubit Pair 3 \\
000 & 110 & 001 & Default Bit \\
001 & 100 & 010 & \\
001 & 110 & 000 & \\
\hline
\end{tabular}

Figure 4.4: Cycle and CS Transmit Position With Three CSs

\begin{tabular}{|c|c|c|c|c|c|c|c|}
\hline 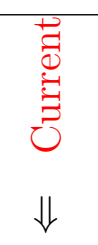 & $\begin{array}{l}E \\
\& \\
8 \\
\Downarrow\end{array}$ & $\begin{array}{l}E \\
\overrightarrow{8} \\
\overrightarrow{8} \\
\Downarrow\end{array}$ & & & & & 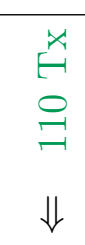 \\
\hline TS1 & TS2 & TS3 & TS4 & TS5 & TS6 & TS7 & TS8 \\
\hline
\end{tabular}




\subsubsection{Four Client Stations}

If it is determined by the AP that there are four CSs wishing to transmit, then the following protocol is used. The AP sends a W-State entangled qubit triad to each of the CSs. They each receive one third of a triad that two others receive for a total of four sets of triads as shown in Figure 4.5.

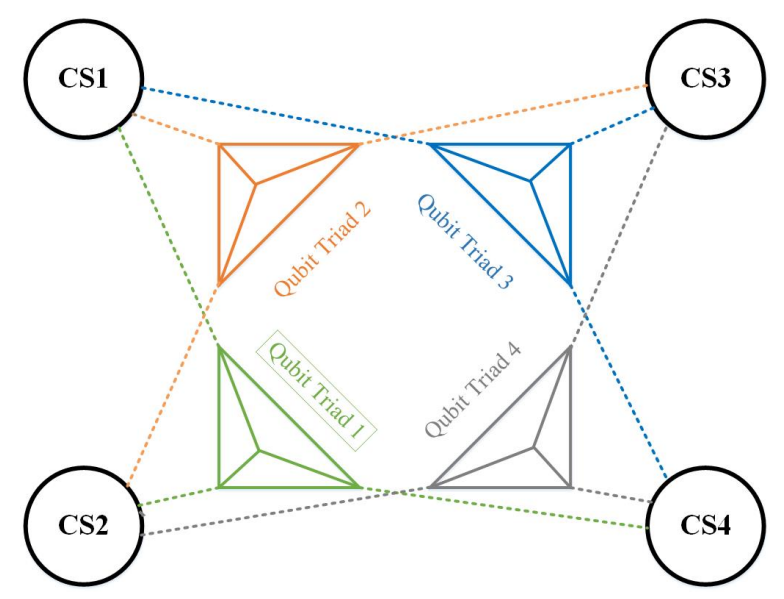

Figure 4.5: Qubit Triad Distribution to Four CSs

As per the entangled protocol, when each triad is read, one of the three is a one, and the other two are zeros. The four qubit triads have a number associated with them from one to four. When the CS reads its qubit from the triad, it puts the result in that position of a four qubit binary number, and then puts a one in the empty slot of that CS. For example, CS4 receives one qubit from triad one, which goes in position one, a second from qubit triad three which goes in the third position, and a third from qubit triad four which goes in its fourth position. The empty position number two receives a one. This leaves the CS with a four qubit binary TN that is unique to itself. This TN tells it how many TSs to wait until it transmits. For example if they have a 0001 they transmit in the first slot from now (TS2). If they have a 0101 they transmit in the fifth slot from now (TS6). See Figure 4.6. Similar to the three CS situation, the "0000" combination does not occur, therefore there are 15 slots available for transmission, of which only four are used by the CSs, leaving 11 for the AP if needed. 
Figure 4.6: Cycle and CS Transmit Position With Four CSs

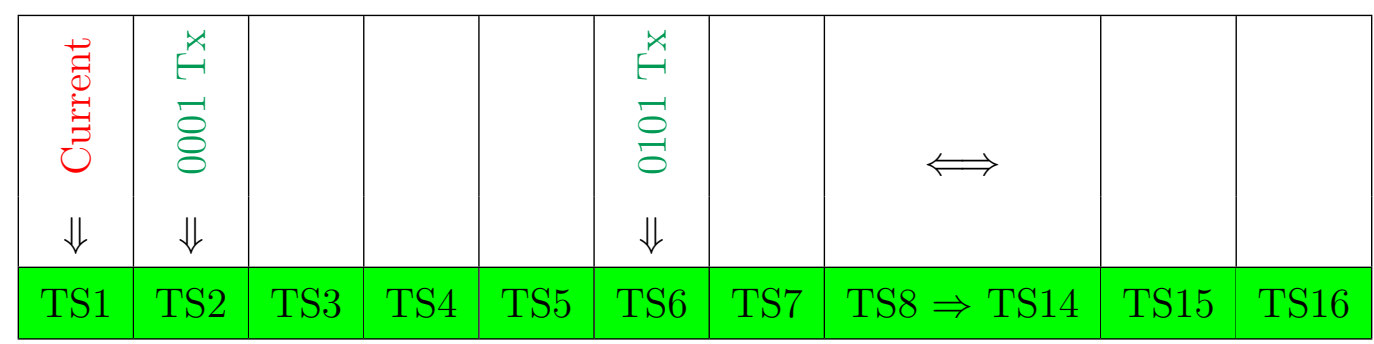

\subsubsection{Unused Time Slots}

In the case where there are three or four CSs wishing to communicate, it has been determined that there are four and eleven empty slots, respectively. Since the AP is unable to know ahead of time what entangled qubit combinations each CS receives, it is unable to know which slots are empty. The AP monitors transmissions during each TS. If after a period of $25 \%$, no transmissions have yet to be received from a CS, it considers this to be an empty slot. The $25 \%$ value gives enough time for a CS to start its transmission if it owns that time slot and leave enough time for the AP to make use of the slot if it is empty. The AP can make use of these empty slots for tasks other than receiving transmissions. It can be used for retransmissions of packets intended for the CS from CSs within the group or from outside the group. It can also use these empty slots to forward packets further in the network outside of the current group. In these cases the packets are half the size of the original and need to be transmitted over two TSs.

\subsection{Communication Protocol Multi-Qubit Entan- glement - Transmit First Election}

As with the previous method, this protocol also achieves $100 \%$ collision avoidance. This is done using W-State entangled qubits. This protocol is similar to a collision resolution using leader election protocol. 
Figure 4.7: Transmission Cycle With One CS

\begin{tabular}{|c|c|}
\hline 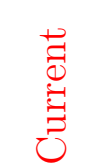 & 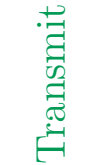 \\
\hline$\Downarrow$ & $\Downarrow$ \\
\hline TS1 & TS2 \\
\hline
\end{tabular}

\subsubsection{One Client Station}

If the AP determines that there is only one CS, then that CS transmits in TS2, and then the process repeats again from the beginning. Refer to Figure 4.7.

\subsubsection{Two Client Stations}

If the AP determines that there are only two CSs wishing to transmit, or there are only two CSs remaining in a group of n CSs, it then sends a pair of W-State entangled bits to them. One half of the pair to each of them. When they receive their qubit, they read it. Based on the principles of entangled pairs, one reads a zero, the other reads a one. This is their Transmit Number (TN). (All possible combinations are shown in Table 4.3.) This TN lets each of them know when to transmit. If the CS reads a zero for the TN, they transmit in the next TS. If they read a one for their TN, they skip the next TS and transmit on the one after that. At the completion of the TSs (which would be three TSs in this case see Figure 4.8), the cycle repeats at the beginning again.

Table 4.3: Two CS Qubit Combinations

\begin{tabular}{|c|c|}
\hline CS1 & CS2 \\
\hline 1 & 0 \\
\hline 0 & 1 \\
\hline
\end{tabular}


Figure 4.8: Cycle and CS Transmit Position With Two Remaining CSs

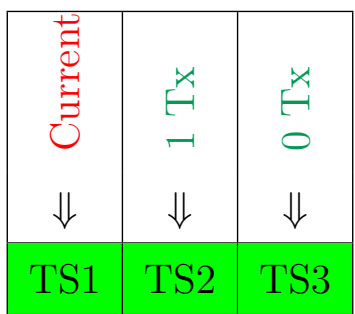

\subsubsection{Three or More Client Stations}

If it has been determined that there are three or more Client Stations (n) wishing to transmit, the AP sends each of them a qubit from a W-State entangled group of $n$ qubits. When they receive their qubit they read it. Based on the W-State entanglement of $n$ qubits, as explained previously, only one of the CSs in the group reads a one, all the others (n-1) read a 0. See table 4.4 for an example of the possible outcomes in the case where $\mathrm{n}=4$ CSs. The CS that receives a one, transmits in the next TS. The remainder of the group repeat the cycle until there are only two of them left at which point they move onto the procedure as explained in the previous section (Two Client Stations).

Table 4.4: Four CS Qubit Combinations

\begin{tabular}{|c|c|c|c|}
\hline CS1 & CS2 & CS3 & CS4 \\
\hline 1 & 0 & 0 & 0 \\
\hline 0 & 1 & 0 & 0 \\
\hline 0 & 0 & 1 & 0 \\
\hline 0 & 0 & 0 & 1 \\
\hline
\end{tabular}

Figure 4.9 shows the total number of TSs that would be required if the round started with four CSs.

\subsubsection{AP Tasks and Joining Transmission Queue}

In this protocol, a complete cycle is when all the CSs have had a chance to transmit. In the case of one CS that is two TSs, three TSs for two CSs, and (2n - 1) TSs for 
Figure 4.9: Total Transmission Cycle With Four CSs

\begin{tabular}{|c|c|c|c|c|c|c|}
\hline 安 & \begin{tabular}{l}
$\mathcal{U}$ \\
0 \\
\multirow{n}{*}{}
\end{tabular} & 安 & $\begin{array}{l}\text { Ĵ } \\
\text { تี } \\
\text { त्र }\end{array}$ & 安 & 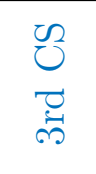 & $\begin{array}{l}\text { 己2 } \\
\text { 靑 }\end{array}$ \\
\hline$\Downarrow$ & $\|$ & $\Downarrow$ & $\Downarrow$ & $\Downarrow$ & $\Downarrow$ & \\
\hline S1 & TS2 & TS3 & TS4 & TS5 & TS6 & $\mathrm{TS}$ \\
\hline
\end{tabular}

any amount greater than two. Since the AP only needs a fraction of time to transmit all the qubits needed in a cycle, this does not add a significant amount of overhead to the cycle. If the AP has tasks that it needs to perform, such as forwarding packets, it does so in between cycles. At that point the AP does not send out any qubits, and just goes ahead with its tasks. It only uses the equivalent of three TSs at this time, to avoid keeping CSs waiting too long to transmit. When completed, it resumes the cycle again. This is also the point at which time the AP reassesses how many CSs are present that wish to transmit.

\subsection{Summary}

With one or two CSs, either protocol uses the same number of TSs to ensure all CSs get to transmit. The savings come when three or four CSs wish to transmit. Table 4.5 shows a comparison of total TSs used to allow all of the CSs the opportunity to transmit. The number in brackets in the Collision Avoidance column refers to the number of unused time slots in that cycle.

Table 4.5: Total Time-slots used Protocol Comparisons

\begin{tabular}{|c|c|c|}
\hline Protocol & Collision Avoidance & Transmit First Election \\
\hline One CS & 2 & 2 \\
\hline Two CSs & 3 & 3 \\
\hline Three CSs & $8(4)$ & 5 \\
\hline Four CSs & $16(11)$ & 7 \\
\hline \hline
\end{tabular}




\section{Chapter 5}

\section{Protocol Testing and Assessment}

There are two types of protocol being used in today's networks. One is non-contention based and the other is contention based. Non-contention based protocol involves algorithms to ensure that when a Client Station (CS) wishes to transmit, there is no other CSs allowed to transmit at the same time. This ensures no collision during transmission. However, these protocols often involve more overhead and thus decreased transmission efficiency. Because of lower overheads and less complicated algorithms, the classic wireless network is often a contention based network such as the Aloha protocol. This means that any computer in the network can transmit data at any time, or in other words a first come-first served protocol. With this type of protocol there are often collisions in transmissions when two or more CSs try to access the AP at the same time. The amount of collisions that occur depend on the network protocol being used. When these collisions occur, the transmissions involved are usually lost or canceled, and have to be retransmitted. The more collisions that occur, the lower the transmission efficiency of the network becomes.

Although our protocols are non-contention based, to ensure full comparison, they are compared to both a non-contention based and a contention based protocol. Due to lack of non-contention based simulation models, simulations of our protocols are only run against a contention based model. The comparison against the non-contention based protocol is done through a analytical process.

\subsection{Non-Contention Based Comparison}

Contention-free Media Access Control (MAC) protocols use algorithms or specialized hardware to ensure collision avoidance. Two of these protocols are one that employs 
a bit-map protocol and another that uses a binary-countdown assignment method. Another third protocol uses a combination of algorithms, software, and a specialized hardware method using assigned transmission frequencies.

\subsubsection{Bit-map Protocol [1]}

In a bit-map protocol there are $\mathrm{n}$ CSs that each have a unique address from 0 to $\mathrm{n}-1$. This protocol is based on a cycle of $\mathrm{n}$ contention slots followed by $\mathrm{n}$ equal transmission TSs. For example, if a CS with address 0 wishes to transmit, it transmits a 1 in the $0^{\text {th }}$ contention slot and the CS that has address 7 , transmits a 1 in the $7^{\text {th }}$ contention slot as in Figure 5.1. No other CSs are allowed to transmit during those time slots. After the cycle has gone through the $\mathrm{n}-1$ contention slots, it begins a transmission cycle of a length equal to the number of CSs that declared their intention in the previous step as in Figure 5.2. The order of transmission is sequential based on the declarations in the contention cycle. When all CSs have finished transmitting, the contention cycle begins again. Since all CSs have a predetermined transmit slot, there are no collisions.

Figure 5.1: Bit-map Contention Slot Transmission Intention

\begin{tabular}{|c|c|c|c|c|c|c|c|c|}
\hline $\begin{array}{c}1 \\
\Downarrow\end{array}$ & & & & & & & $\begin{array}{c}1 \\
\Downarrow\end{array}$ & $\Longleftrightarrow$ \\
\hline TS0 & TS1 & TS2 & TS3 & TS4 & TS5 & TS6 & TS7 & TS8 $\Rightarrow$ TSn-1 \\
\hline
\end{tabular}

Figure 5.2: Bit-map Transmission Slots

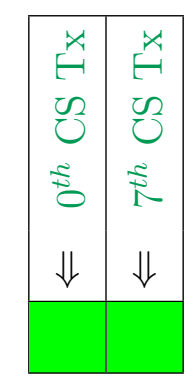

This protocol has the overhead equal to the number of slots needed for the contention slot length n-1. Even it all CSs do not wish to transmit, a contention slot is 
allotted for them. So if there are ten CSs present but only two wish to transmit, then eight contention slots are wasted. With our TFE protocol, there are no wasted slots. All slots are used for data transmission or entangled qubit transmission. With the CA protocol, there is the possibility of a number of wasted TSs, of a length greater than this protocol.

\subsubsection{Binary-countdown Assignment Protocol [1]}

All CSs are assigned a fixed length binary address in binary count down protocol. If a CS wishes to transmit, it first broadcasts its address as a binary bit string, starting with its Most Significant Bit (MSB) (left to right). All the different CSs wishing to transmit have their binary addresses logically OR-ed together to decide the priority of transmitting. For example, (refer to Table 5.1), if four CSs trying to transmit have addresses of 0010, 0100, 1010, and 1001, they first transmit their address MSB, i.e., 0, 0, 1,1 respectively. These bits are OR-ed resulting in the first two stations being dropped from this current round. The other two stations continue since they both have a 1 as their MSB. The next bit for the remaining two stations are both 0 , so they move on the third bit. The third bits are OR-ed together resulting in station 1001 being dropped, and station 1010 having the highest address and therefore the right to transmit first. This cycle then repeats for the remaining CSs wishing to transmit until all CSs have an order they transmit in. They then transmit in order based on these results. After all CSs have transmitted, then the cycle repeats with all CSs wishing to transmit.

Table 5.1: Binary Count Down Protocol

\begin{tabular}{|c|c|}
\hline $\begin{array}{c}\text { Station } \\
\text { address }\end{array}$ & $\begin{array}{c}\text { Bit time } \\
0123\end{array}$ \\
\hline 0010 & $0-$ \\
0100 & $0-$ \\
1010 & 1010 \\
1001 & $100-$ \\
\hline
\end{tabular}

With this protocol, the overhead involved is the algorithm processing the order of transmission. Also, the CSs with the highest binary address always transmit first. In 
our protocols, the order of transmission are always determined at the time of reading the entangled bit, so no calculation is needed. The TFE and CA protocols also are completely random, therefore allowing for equal chance at transmitting first.

\subsubsection{Assigned Transmission Frequencies Protocol}

In this protocol, the Access Point (AP) uses a common frequency that is available to all CSs for it to send out communications. In turn each of the CSs have their own individual frequency to transmit back to the AP. This protocol produces $100 \%$ collision avoidance. This protocol requires that each CS be assigned their own bandwidth when they join the network. To do this they must have the hardware or software that allows them to change frequencies at will. This leads to added costs and overhead for that CS. The AP requires hardware and software that allows them to handle and process the incoming and outgoing communications in parallel. This also requires an added cost and overhead as well.

Our protocols would need hardware to send and receive qubits and the required software to run them. Once installed the overhead would be at a minimum. At present this hardware is costly but will be reduced with mass production and product evolution.

\subsubsection{Non-Contention Based Comparison Results}

In theory the TFE protocol performs just as good if not better than bit-mapping and binary count-down protocols. This is due to less overhead needed by the TFE protocol over that of the other two. When you compare the TFE protocol to the one that uses assigned transmission frequencies ours has less overhead, but looses out on the cost of needed hardware. With future hardware developments this should no longer be the case and therefore not an issue at that time. The CA protocol on the other hand does not do as well as these protocols because of its empty slots.

\subsection{Contention Based Simulation Comparison}

Since most of the technology that is needed to run a wireless network using Quantum Assisted MAC protocol is still in development, it is necessary to use simulation software to conduct tests on this type of network. For this simulation and comparison 
of our protocols, we use OMNeT $++[25]$. OMNeT ++ is a simulation library and framework, primarily for building network simulators using modular and componentbased $\mathrm{C}++$. The two networks simulating the quantum protocols were written from scratch. These are being compared to a standard existing network protocol, Aloha which can be found within $\mathrm{OMNeT}++$.

The Aloha simulation shows only the transmissions from clients to the access point. It does not show any forwarding of any messages to the clients inside or outside of the network. There are also no messages coming in from outside of the network. Therefore, the simulations of our protocols are based on the same principal for ease of comparison and evaluation.

\subsubsection{Qubit Entanglement Simulation}

In both of the network simulations, the actual transmission and reading of entangled qubits are simulated using random number generation algorithms. All qubit values received by the CSs are random following the proposed protocols. In the CA network, the qubit transmit number combinations received by each CS is unique to the device receiving it. In the TFE network, only one device in each AP transmission round receives a one, all others receive a zero as per the protocol.

\subsubsection{Architecture}

For the Collision Avoidance protocol network, the simulation is based on one access point placed within the transmission area, with four locations at random points surrounding the AP as in Figure 5.3. These client stations can be anywhere within the AP service area, as long as they follow the guidelines of quantum communication qubit transmission protocols. Although this protocol can be used for one to four CSs, for simulation purposes, four was chosen.

The Transmit First Election protocol network is similar. The simulation is based on one access point placed within the transmission area, with six locations at random points surrounding the AP as in Figure 5.4. These client stations can be anywhere within the AP service area, as long as they follow the guidelines of quantum communication qubit transmission protocols. Although this protocol can be used for $n$ number of CSs, for simulation purposes, six was chosen. 


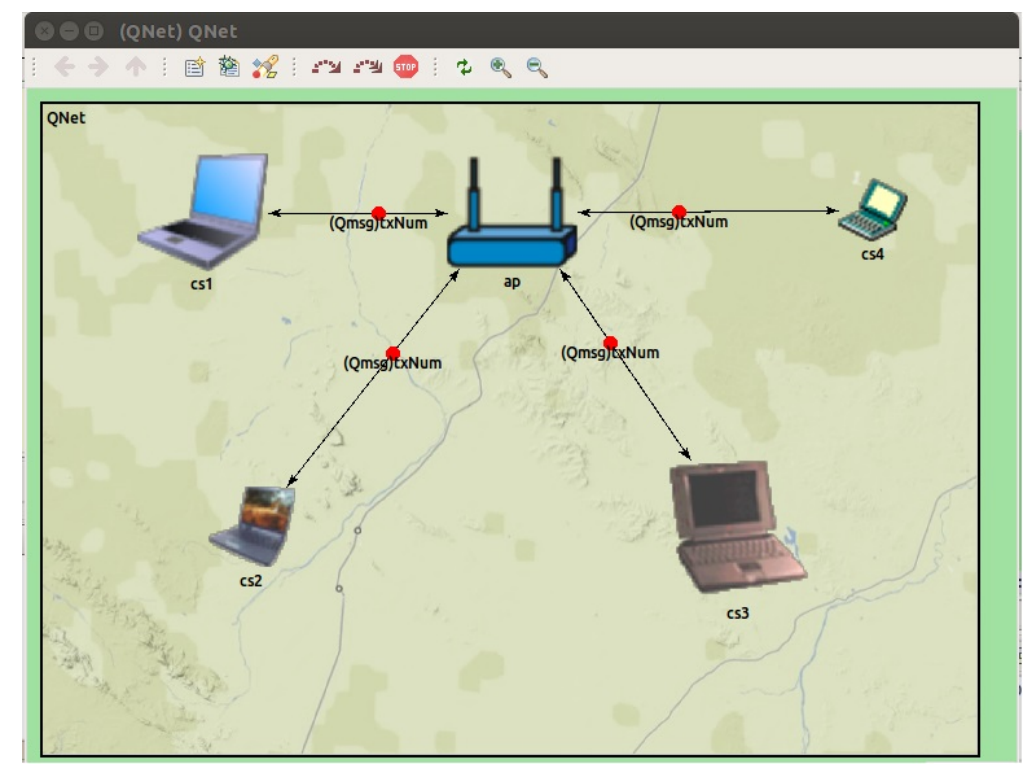

Figure 5.3: Architecture of Collision Avoidance Network

In either of the above networks, the client stations can be of any type of wireless network communication device, such as laptop or tablet, that can be equipped with a device for receiving qubits. Once the protocol has determined when the device is allowed to transmit, it uses classic wireless protocols for transmission.

\subsubsection{Event Based}

To test these protocols a discrete-event based simulation is being used. When using simulations, discrete-event simulations are often used to model the operation of a system as a discrete sequence of events over a period of time. During the period of time, any events occur at a certain moment in time as a change in state to that system. The simulation can move straight from one event to the next consecutive one, since no change in the system is assumed to have happened.

In this simulation, the AP transmits the entangled qubits to all the CSs involved. In the CA protocol these entangled qubits allow the CSs to receive their Transmit Numbers. This TN then triggers and schedules an event for each given CS to transmit its packet at the proper time slot in the cycle. For the TFE protocol, these entangled qubits trigger and schedule one CS to transmit in the next time slot in the cycle. 


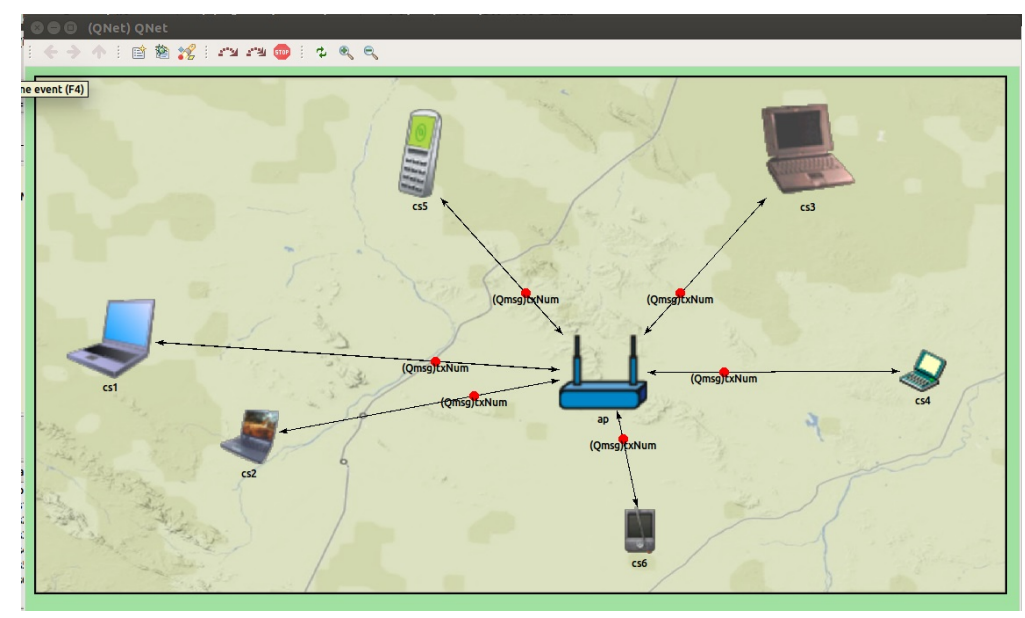

Figure 5.4: Architecture of Transmit First Election Network

\subsubsection{Protocol Testing}

My two protocols are tested by comparing them against the current Slotted Aloha protocol. In the Aloha protocol simulation, the Server (AP) only receives transmissions from the hosts and does not forward these transmissions on to their intended destination, nor does it receive transmissions from internal and external sources destined for the hosts within the current network. To keep things on an even comparison, my protocols are tested in the same manner. That is, the AP only transmits the necessary entangled bits, and not forward on any transmission of packets.

The Aloha simulation was adjusted to have four hosts (see Figure 5.5) instead of the preprogrammed 20 to make fairer comparisons against the CA protocol that also used four hosts (see Figure 5.6). The Aloha simulation uses randomization to select which hosts tries to transmit at any given time, and was allowed to run for a period of time to get average observation values. Since, the CA protocol repeats a cycle of the same number of time slots, the simulation was run a few cycles to obtain observation values. This repeating of cycles was also done to ensure that the selection of CS transmissions appeared to be random as based on the described protocol, and each CS had a chance to transmit.

Next the Aloha simulation was adjusted to have six hosts (see Figure 5.7) instead of the preprogrammed 20 to make fairer comparisons against the TFE protocol that 


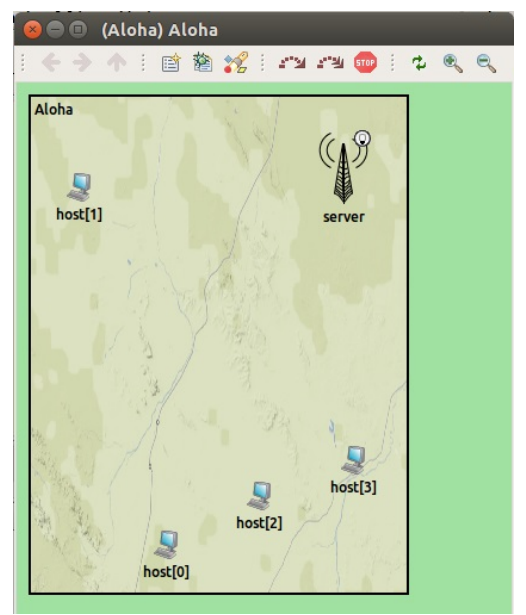

Figure 5.5: Aloha Simulation With 4 Hosts

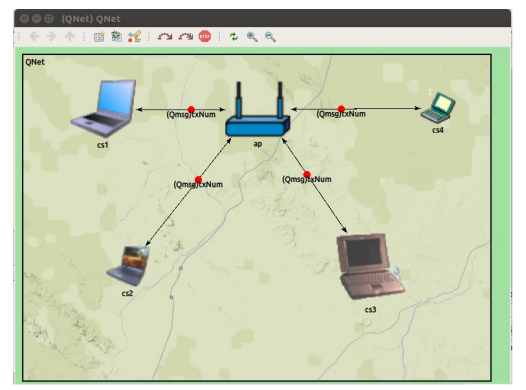

Figure 5.6: Collision Avoidance Simulation With 4 Hosts

also used six hosts (see Figure 5.8). As previously, the Aloha simulation uses randomization to select which host tries to transmit at any given time, and was allowed to run for a period of time to get average observation values. The TFE protocol simulation was run for the same amount of time to obtain observation values. Allowing the simulation to run for a period of time was also done to ensure that the selection of host transmissions appeared to be random as based on the described protocol, and each CS had a chance to transmit in a timely and fair manner.

\subsubsection{Contention Based Simulation Comparison Results}

When the CA and TFE protocol simulations were run, they behaved exactly as expected. During each cycle of the CA simulation run, each of the CSs were able to transmit once within a cycle, and appeared to be random from one cycle to the next. 


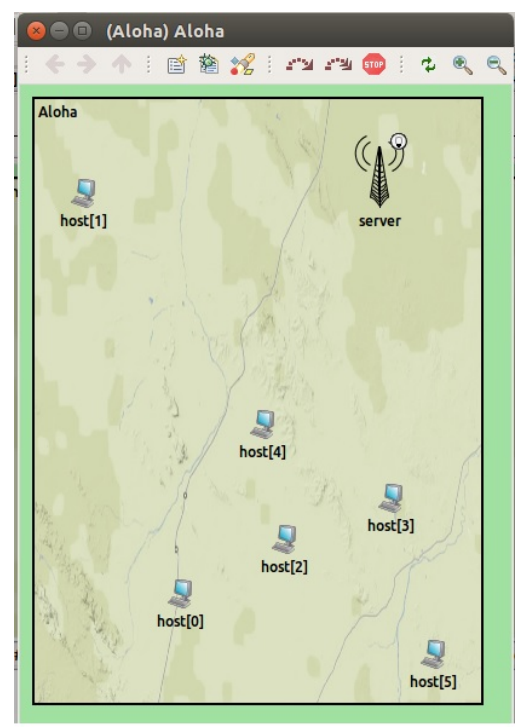

Figure 5.7: Aloha Simulation With 6 Hosts

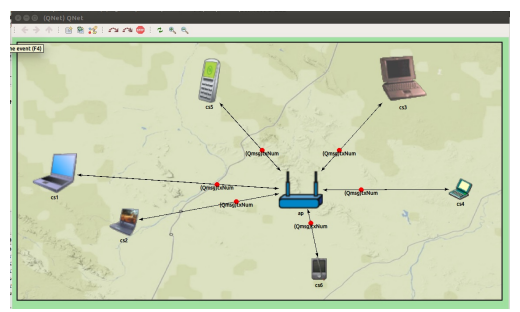

Figure 5.8: Transmit First Election Simulation With 6 Hosts

As a result of this, there were no collisions reported throughout the simulation. The TFE simulation was successful as well. Each of the CSs was given a chance to transmit within a fair and timely fashion as per the protocols. Over the period of the simulation, the order of who Transmitted First was random as per the protocols, and no collisions were reported throughout the simulation. When it came to the Aloha simulations, there were collisions in both runs as expected.

For comparison stats, the effective transmission values were calculated for all the various versions of the Aloha Protocols. First the Effective Data Rate (EDR) was calculated using Formula 5.1. This value is calculated by first subtracting the collisions observed (Collisions) from the number of packets received (Redc Pks) during a given period. It is then divided by the length of time of the observation period (Duration). This result is then multiplied by the packet length in bits (pkLenBits) to achieve the EDR. 


$$
E D R=\frac{\text { RecdPks }- \text { Collisions }}{\text { Duration }} \times p k \text { LenBits }
$$

The calculated EDR is then used in the Transmission Efficiency (TxEff) Formula 5.2 to give us an Transmission Efficiency value. To calculate the TxEff, you divide the previously calculated EDR by the transmission rate (TxRate) and then multiply it by 100 percent.

$$
T x E F F=\frac{E D R}{\text { TxRate }} \times 100 \%
$$

The transmission efficiency of the different Aloha protocols, as per the previous two calculations, can be seen in Figure 5.9. There are three states of two configurations, giving six different results of the Aloha protocol shown here.

The two configurations are Pure and Slotted. In the slotted Aloha protocol, the transmitting station always broadcasts at the full rate of the channel. This transmission starts at the beginning of a slot. A collision occurs if two or more stations transmit at the same time. All the stations involved in this collision try to retransmit the frame at random intervals until a successful delivery is achieved. This requires all the stations to be synchronized to the slot timings. The non-slotted Aloha protocol, known as Pure, does not use slot synchronization. A station that wishes to transmit, does so when it wants to. In this case a collision can occur at any time during the transmission of the packet. Since there are no slots, a station may start to transmit, when another one is already in the process of transmitting its frame causing the collision. If it does, than as before the stations involved try to retransmit at a random interval until success has occurred.

The three states of the protocols are overloaded(1), optimal load(2), and low traffic(3). The overloaded state uses too frequent transmissions that result in high collision rate and low channel utilization. In the optimal load state, near optimal load channel utilization is near theoretical maximum $1 / 2$ e. The low traffic state uses very low traffic that results in channel being idle most of the time.

As can be seen from the chart (Figure 5.9) of the various Aloha protocol combinations, the Slotted Aloha 2 is the most efficient of that group. Since this is the upper end of the transmission efficiency for the Aloha protocols, this is the protocol 


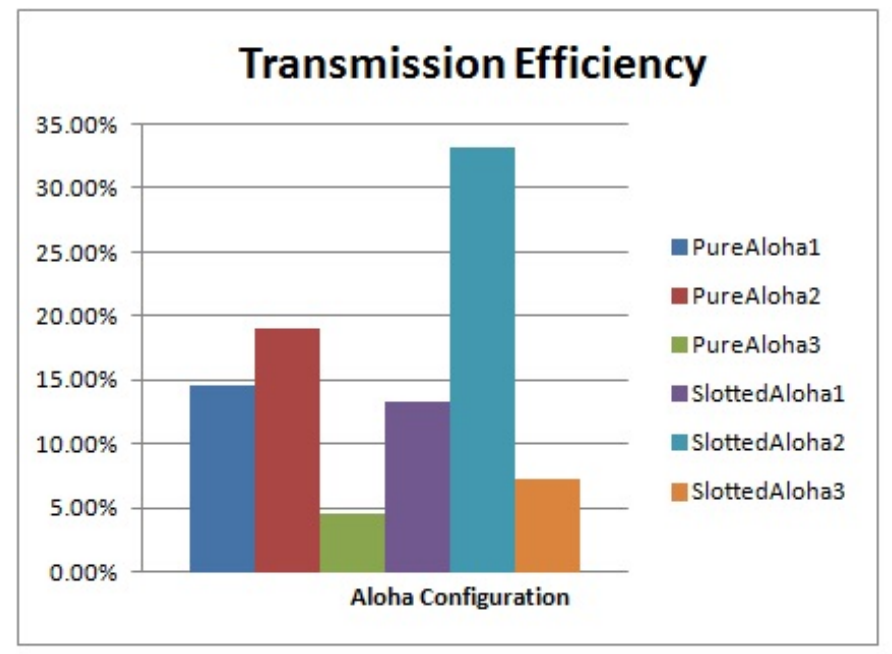

Figure 5.9: Transmission Efficiency for Aloha Protocols

that was used to compare against our protocols.

Table 5.2: Transmission Efficiency Comparisons

\begin{tabular}{|c|c|c|c|c|}
\hline Protocol & Packets Received & Collisions & Effective Rate (bps) & Tx Efficiency \\
\hline CA & 156 & 0 & 2475.2 & $25.78 \%$ \\
\hline TFE & 576 & 0 & 9139.2 & $95.2 \%$ \\
\hline Aloha & 546 & 345 & 3189.2 & $33.22 \%$ \\
\hline \hline
\end{tabular}

\begin{tabular}{|c|c|}
\hline \hline Event Time & $60 \mathrm{~s}$ \\
\hline Packet Size (bits) & 952 \\
\hline Tx Rate (bps) & 9600 \\
\hline \hline
\end{tabular}

Using the afore mentioned formula and the values obtained during the simulation, Effective Data Rate and Transmission Efficiency were calculated for our protocols. A comparison of these results, along side the Slotted Aloha 2, can be seen in Table 5.2 for comparison.

The transmission efficiency for the CA protocol was below that of the Slotted Aloha 2, but well above all the other Aloha protocols. This is largely due to the empty slots that are in the cycle of the CA protocol. As these slots are used by the AP to conduct other AP tasks, the transmission efficiency of this protocol increases. Table 5.3 shows what the transmission efficiency should be if there are one, two, or 
three CSs using the CA protocol. The transmission efficiency for one and two CSs is higher because there are no empty slots in the cycle for these cases. With three CSs there are 4 empty slots within the cycle causing lower efficiency, but as with four, these slots can be used by the AP for other tasks to raise the efficiency.

Table 5.3: Calculated Transmission Efficiencies For Other Numbers of CSs

\begin{tabular}{|c|c|c|c|c|}
\hline Protocol & Packets Received & Collisions & Effective Rate (bps) & Tx Efficiency \\
\hline CA 1 CS & 571 & 0 & 9059.87 & $94.37 \%$ \\
\hline CA 2 CS & 584 & 0 & 9266.13 & $96.52 \%$ \\
\hline CA 3 CS & 255 & 0 & 4046 & $42.15 \%$ \\
\hline TFE 1 & 571 & 0 & 9059.87 & $94.37 \%$ \\
\hline TFE 10 & 574 & 0 & 9107.47 & $94.87 \%$ \\
\hline TFE 48 & 571 & 0 & 9059.87 & $94.37 \%$ \\
\hline \hline
\end{tabular}

\begin{tabular}{|c|c|}
\hline \hline Event Time & $60 \mathrm{~s}$ \\
\hline Packet Size (bits) & 952 \\
\hline Tx Rate (bps) & 9600 \\
\hline
\end{tabular}

When it came to the transmission efficiency for the TFE protocol, it far surpassed that of the Aloha protocols (table 5.2). Also, as can be seen in table 5.3, the transmission efficiency stays relatively the same regardless of how many CSs there are, up to 48. (48 CSs is the upper bound based on the TFE protocol, since the AP can only transmit up to 48 bits in it's $5 \mathrm{~ms}$ of transmit time.)

In both protocol cases, CA and TFE, the transmission efficiency can never reach $100 \%$. This is because of the overhead that is required by the AP. The AP uses slots of $5 \mathrm{~ms}$ to transmit the entangled qubits and monitor the transmissions of the CSs. The CA protocol only requires one of these time slots per cycle, but the TFE uses $\mathrm{n}$ (number of CSs transmitting) during each full cycle, so as the $\mathrm{n}$ increases, so does the overhead, thus allowing the transmission efficiency to never reach $100 \%$.

\subsubsection{Statistical Analysis of Data}

In this section, $\alpha$ is the level of significance, which is $5 \%$ in our calculations. This in turn corresponds to a confidence level of $\mathrm{P}=100(1-\alpha) \%$. Therefore our confidence 
level is $95 \%$ or inversely stated as an error rate of $5 \%$.

To determine the confidence level of the data, we first conducted an F-Test using MS-Excel to determine if the results are of equal or unequal variance. Since in the two comparisons (see Tables 5.4, 5.5) $\mathrm{P}<0.05$, the variances are unequal.

Table 5.4: F-Test Aloha vs TFE

\begin{tabular}{|c|c|c|}
\hline & Aloha & TFE \\
\hline Mean & 0.15315 & 0.947025 \\
\hline Variance & 0.01036 & $1.6556 \mathrm{E}-05$ \\
\hline Observations & 6 & 4 \\
\hline $\mathrm{df}$ & 5 & 3 \\
\hline$P(F \leq f)$ one-tail & 0.000010 & \\
\hline \hline
\end{tabular}

Table 5.5: F-Test Aloha vs CA

\begin{tabular}{|c|c|c|}
\hline & Aloha & CA \\
\hline Mean & 0.15315 & 0.64705 \\
\hline Variance & 0.01036 & 0.13054 \\
\hline Observations & 6 & 4 \\
\hline $\mathrm{df}$ & 5 & 3 \\
\hline$P(F \leq f)$ one-tail & 0.00910 & \\
\hline \hline
\end{tabular}

Based on the F-Test results we conduct a T-Test: two-tail two sample assuming unequal variances using an $\alpha$ of 0.05 .

Examining the data comparison between the Aloha protocol and the TFE protocol (see Table 5.6 ) we cannot reject the Null Hypothesis because $\mathrm{p}<0.05$. Therefore the observed difference between the data is convincing enough to say that the average Tx Efficiency of TFE over Aloha differs significantly.

Examining the data comparison between the Aloha protocol and the CA protocol (see Table 5.7 ) we reject the Null Hypothesis because $\mathrm{p}>0.05$. Therefore the 
observed difference between the data is not convincing enough to say that the average Tx Efficiency of CA over Aloha differs significantly.

Table 5.6: T-Test Aloha vs TFE

\begin{tabular}{|c|c|c|}
\hline & Aloha & TFE \\
\hline Mean & 0.15315 & 0.947025 \\
\hline Variance & 0.01036 & $1.6556 \mathrm{E}-05$ \\
\hline Observations & 6 & 4 \\
\hline Hypothesized Mean Difference & 0 & \\
\hline$P(F \leq t)$ two-tail & $7.28993 \mathrm{E}-06$ & \\
\hline \hline
\end{tabular}

Table 5.7: T-Test Aloha vs CA

\begin{tabular}{|c|c|c|}
\hline & Aloha & CA \\
\hline Mean & 0.15315 & 0.64705 \\
\hline Variance & 0.01036 & 0.13054 \\
\hline Observations & 6 & 4 \\
\hline Hypothesized Mean Difference & 0 & \\
\hline$P(F \leq t)$ two-tail & 0.07605 & \\
\hline \hline
\end{tabular}




\section{Chapter 6}

\section{Conclusions and Future Work}

Ever since the first electromechanical analog computer was built by the United States Navy in 1938, we have been trying to improve speed, versatility, and connect-ability in computers. Since the 1970s and 1980s quantum mechanics has become a part of these improvement scenarios. This led to theoretical quantum computers and quantum communication. In the latter part of the 20th century and the beginning of the 21st century, quantum communication has moved from the theoretical world to the physical world.

The ability to transmit a quantum state from one location to another is the basis of quantum communication. Ever increasing numbers of qubit entanglement, increased wireless transmission distances, and quantum networking is being realized. These improvements have been always carried out between two entities. It is time to have classic multi-user computing networks take advantage of quantum communication.

In classic wireless communication the access point allows many client stations (CS) to communicate with it as part of a network. The Medium Access Control protocol handles management of the communication to these access points (AP). Quantum networking also needs this type of protocol to take it from one-to-one communications to multi-user network communications. Most classic wireless networks use a contention based network protocol. This protocol allows computers within a network to communicate with the access point on a first come first served basis. The downside of this protocol is that collisions in transmissions can occur decreasing the overall transmission efficiency of the network.

This thesis has examined two quantum assisted MAC protocols that take advantage of quantum entanglement principles to aid classic networking. These two protocols allow for multiuser access to a communication network. With the use of 
quantum entanglement, a contention-free collision avoidance technique is used. This enables a cycle of time slots which enable the client stations to have equal and fair access to the access point. The collision avoidance protocol cycles vary in length depending on the number of CSs present. In this protocol, the number of time slots needed in one cycle is 2 for $1 \mathrm{CS}, 3$ for $2 \mathrm{CSs}, 8$ for $3 \mathrm{CSs}$ and 16 for $4 \mathrm{CSs}$. The second protocol is a transmit first election type collision avoidance protocol. For this protocol, the number of time slots needed for a complete cycle is $2 n-1$, where $n$ is the number of CSs present wishing to transmit.

When the proposed protocols were compared against some of the existing contention-free protocols, the TFE came out on top. With its lower overhead and built in randomization, it performs better than the other protocols looked at. The CA protocol did not fair as well, due to wasted TSs.

Since portable low cost quantum transmission devices are not available, physical testing of the protocols was not possible therefore network simulation software had to be used to test these protocols. To move ahead on the Quantum Communication assisted MAC protocols, it will have to be physically tested in a network. This will be possible when $\mathrm{R} \& \mathrm{D}$ produces lower costing hardware to make the testing more cost efficient and viable.

The network simulations show that these protocols preform just as good as contention based Aloha slotted protocol. The transmission efficiency for the CA protocol was below that of the Slotted Aloha 2, but well above all the other Aloha protocols. This is largely due to the empty slots that are in the cycle of the CA protocol. As these slots are used by the AP to conduct other AP tasks, the transmission efficiency of this protocol increases. When it came to the transmission efficiency for the TFE protocol, it far surpassed that of the Aloha protocols. With this protocol, the transmission efficiency stays relatively the same regardless of how many CSs there are present.

To move ahead with these protocols, changes will have to be made to the current MAC layer. A sub-layer that is designed to handle quantum protocol will have to be added. With the addition of the sub layer, the current MAC layer will be able to control both classic and quantum communication protocol. For future development, there also needs to be low cost quantum routers and implementation of quantum transceivers within portable devices such as laptops, computers, and tablets. These devices will allow for full use of the protocols proposed here, as well as the transmission 
of data quantumly instead of the current WiFi technology.

With the use of the protocols presented here the advancement of classic networking efficiency can occur. With further development of quantum transmissions, changes to the MAC layer and the use of these protocols, full quantum networks cannot be far off. 


\section{List of References}

[1] I. Dhotre, Data Communication. Technical Publications, 2007.

[2] M. Berces and S. Imre, "Modeling Medium Access Control by Quantum Methods," INES 2006, 10th International Conference on Intelligent Engineering Systems, June 2006.

[3] C. M. Arizmendi, J. P. Barrangu, and O. G. Zabaleta, "A 802.11 MAC Protocol Adaptation for Quantum Communications," 2012 IEEE/ACM 16th International Symposium on Distributed Simulation and Real Time Applications, October 2012.

[4] M. A. Nielsen and I. L. Chuang, Quantum Computation and Quantum Information. Cambridge University Press, 2010.

[5] J. Gribbin, Computing With Quantum Cats : From Colossus to Qubits. London : Bantam, 2013.

[6] R. V. Meter, Quantum Networking. Hoboken NJ, Wiley, 2014.

[7] W. Dur, G. Vidal, and J. I. Cirac, "Three Qubits Can be Entangled in Two Inequivalent Ways," A Physical Review, vol. 62, June 2000.

[8] D. M. Greenberger, M. A. Horne, and A. Zeilinger, "Going Beyond Bells Theorem," 1989.

[9] WikiMedia, "http://www.wikipedia.org/," 2015. [Accessed Online; Sept 2014 Mar 2015].

[10] A. Einstein, B. Podolsky, and N. Rosen, "Can Quantum-mechanical Description of Physical Reality be Considered Complete?," Physical review, vol. 47, no. 10, p. $777,1935$.

[11] J. Bell, "On The Einstein Podolsky Rosen Paradox," Physics, vol. 1, November 1964.

[12] F. Verstraete, J. Dehaene, B. D. Moor, and H. Verschelde, "Four Qubits Can be Entangled in Nine Different Ways," A Physical Review, vol. 65, March 2002.

[13] T. Monz, P. Schindler, J. T. Barreiro, M. Chwalla, D. Nigg, W. A. Coish, M. Harlander, W. Hnsel, M. Hennrich, and R. Blatt, "14-qubit Entanglement: Creation and Coherence," Physical Review and Letters, vol. 106, March 2011. 
[14] H. Bennett Ch and G. Brassard, "Quantum Cryptography: Public Key Distribution and Coin Tossing," pp. 175-9, 1984.

[15] M. Sasaki, M. Fujiwara, H. Ishizuka, W. Klaus, K. Wakui, M. Takeoka, S. Miki, T. Yamashita, Z. Wang, A. Tanaka, et al., "Field Test of Quantum Key Distribution in the Tokyo QKD Network," Optics Express, vol. 19, no. 11, pp. 1038710409, 2011.

[16] L. O. Mailloux, M. R. Grimaila, D. D. Hodson, G. Baumgartner, and C. McLaughlin, "Performance Evaluations of Quantum Key Distribution System Architectures," IEEE Security $\&$ Privacy, no. 1, pp. 30-40, 2015.

[17] R. Ursin, F. Tiefenbacher, T. Schmitt-Manderbach, H. Weier, T. Scheidl, M. Lindenthal, and B. B. et al., "Entanglement-based Quantum Communication Over 144 km," Nature Physics, vol. 3, July 2007.

[18] C.-Z. Peng, T. Yang, X.-H. Bao, J. Zhang, X.-M. Jin, F.-Y. Feng, B. Yang, J. Yang, J. Yin, Q. Zhang, et al., "Experimental Free-space Distribution of Entangled Photon Pairs Over 13 km: Towards Satellite-based Global Quantum Communication," Physical review letters, vol. 94, no. 15, p. 150501, 2005.

[19] C. H. Bennett, G. Brassard, C. Crépeau, R. Jozsa, A. Peres, and W. K. Wootters, "Teleporting an Unknown Quantum State Via Dual Classical and EinsteinPodolsky-Rosen Channels," Physical review letters, vol. 70, no. 13, p. 1895, 1993.

[20] S. Singhal, A. Jain, A. K. Grankotiya, and K. Aggarwal, "An Investigation of Quantum Teleportation," 2012 Second International Conference on Advanced Computing and Communication Technologies, January 2012.

[21] R. V. Meter, J. Touch, and C. Horsman, "Recursive Quantum Repeater Networks," Progress in Informatics, May 2011.

[22] J. Touch, Y.-S. Wang, and V. Pingali, "A Recursive Network Architecture," ISI, Tech. Rep, no. 2006-626, 2006.

[23] L. Aparicio, R. V. Meter, and H. Esaki, "Protocol Design for Quantum Repeater Networks," AINTEC 11 Proceedings of the 7th Asian Internet Engineering Conference, November 2011.

[24] R. V. Meter, "Quantum Networking and Internetworking," IEEE Networking, vol. 26, July 2012.

[25] OpenSimLtd, "OMNeT++ Discrete Event Simulator," http: // www. omnetpp . org/, 2015. 


\section{Appendix A}

\section{Acronyms}

$\begin{array}{ll}\text { AP } & \text { Access Point } \\ \text { bps } & \text { bits per second } \\ \text { CA } & \text { Collision Avoidance } \\ \text { CS } & \text { Client Station } \\ \text { EDR } & \text { Effective Data Rate } \\ \text { EPR } & \text { Einstein Podolsky Rosen } \\ \text { ESC } & \text { Entangelment Swapping Control } \\ \text { GHZ } & \text { Greenberger Horne Zeilinger } \\ \text { GHz } & \text { Gigahertz } \\ \text { km } & \text { kilometer } \\ \text { LOCC } & \text { Local Operations and Classical Communication } \\ \text { MAC } & \text { Medium Access Control Protocol } \\ \text { ms } & \text { millisecond } \\ \text { MSB } & \text { Most Significant Bit } \\ \text { OSI } & \text { Open Systems Interconnection } \\ \text { OTP } & \text { One Time Pad } \\ \text { PC } & \text { Purification Control } \\ \text { QKD } & \text { Quantum Key Distribution } \\ \text { R\&D } & \text { Research and Development } \\ \text { SLOCC } & \text { Stochastic Local quantum Operations assisted by Classical Communication } \\ \text { SPDC } & \text { Spontaneous Parametric Down Conversion } \\ \text { TDMA } & \text { Time Division Multiple Access } \\ \text { TFE } & \text { Transmit First Election } \\ \text { TN } & \text { Transmit Number } \\ \text { TS } & \text { Time Slot } \\ \end{array}$

